\title{
a-Synucleinopathy associated c-Abl activation causes p53-dependent autophagy impairment
}

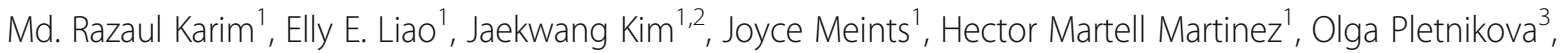
Juan C. Troncoso ${ }^{3}$ and Michael K. Lee ${ }^{1, *^{*}}$ (D)

\begin{abstract}
Background: Studies link c-Abl activation with the accumulation of pathogenic a-synuclein (aS) and neurodegeneration in Parkinson's disease (PD). Currently, c-Abl, a tyrosine kinase activated by cellular stress, is thought to promote aS pathology by either directly phosphorylating aS or by causing autophagy deficits.

Methods: aS overexpressing transgenic (Tg) mice were used in this study. A53T Tg mice that express high levels of human mutant A53TaS under the control of prion protein promoter. Two different approaches were used in this study. Natural aging and seeding model of synucleinopathy. In seeding model, intracortical/intrastriatal (IC/IS) stereotaxic injection of toxic lysates was done using tissue lysates from end-stage symptomatic mice. In this study, nilotinib and pifithrin-a was used as a c-Abl and p53 inhibitor, respectively. Both Tg and non-transgenic (nTg) mice from each group were subjected to nilotinib $(10 \mathrm{mg} / \mathrm{kg})$ or vehicle (DMSO) treatment. Frozen brain tissues from PD and control human cases were analyzed. In vitro cells study was implied for c-Abl/p53 genetic manipulation to uncover signal transduction.
\end{abstract}

Results: Herein, we show that the pathologic effects of c-Abl in PD also involve activation of p53, as c-Abl activation in a transgenic mouse model of a-synucleinopathy (TgA53T) and human PD cases are associated with the increased p53 activation. Significantly, active p53 in TgA53T neurons accumulates in the cytosol, which may lead to inhibition of autophagy. Thus, we hypothesized that c-Abl-dependent p53 activation contributes to autophagy impairment in a-synucleinopathy. In support of the hypothesis, we show that c-Abl activation is sufficient to inhibit autophagy in p53-dependent manner. Moreover, inhibition of either c-Abl, using nilotinib, or p53, using pifithrin-a, was sufficient to increase autophagic flux in neuronal cells by inducing phosphorylation of AMP-activated kinase (AMPK), ULK1 activation, and down-regulation of mTORC1 signaling. Finally, we show that pharmacological attenuation of c-Abl activity by nilotinib treatment in the TgA53T mouse model reduces activation of p53, stimulates autophagy, decreases accumulation as pathology, and delays disease onset.

Conclusion: Collectively, our data show that c-Abl activation by a-synucleinopathy causes p53 dependent autophagy deficits and both c-Abl and p53 represent therapeutic target for PD.

Keywords: Neurodegeneration, Parkinson's disease (PD), a-Synuclein, C-Abl, p53, Autophagy, mTOR, AMPK, Nilotinib, Pifithrin-a

\footnotetext{
* Correspondence: mklee@umn.edu

'Department of Neuroscience, University of Minnesota, Minneapolis, MN

55414, USA

${ }^{4}$ Institute for Translational Neuroscience, University of Minnesota, 2101 6th

Street SE, Minneapolis, MN 55414, USA

Full list of author information is available at the end of the article
}

(c) The Author(s). 2020 Open Access This article is distributed under the terms of the Creative Commons Attribution 4.0 International License (http://creativecommons.org/licenses/by/4.0/), which permits unrestricted use, distribution, and reproduction in any medium, provided you give appropriate credit to the original author(s) and the source, provide a link to the Creative Commons license, and indicate if changes were made. The Creative Commons Public Domain Dedication waiver (http://creativecommons.org/publicdomain/zero/1.0/) applies to the data made available in this article, unless otherwise stated. 


\section{Background}

Parkinson's disease (PD) is a common late onset progressive neurodegenerative disease most characterized by movement disorder resulting from the loss of dopaminergic (DAergic) neurons in the substantia nigra pars compacta (SNpc) [1, 2]. In addition, PD is also characterized by the presence of protein inclusions known as Lewy bodies (LB) and Lewy neurites (LN), which are composed of aggregated $\alpha$-synuclein $(\alpha S)$, in multiple neuronal populations [3-5]. While the etiology of PD is unknown in most cases, $\alpha \mathrm{S}$ abnormalities are mechanistically linked to PD pathogenesis as mutations in $\alpha S$ cause PD in a small number of familial PD pedigrees [6, 7]. Currently, how $\alpha \mathrm{S}$ abnormalities cause neuronal dysfunction and degeneration is not fully understood. However, studies have implicated oxidative stress in the pathogenesis of PD [8-10] and dysfunction in proteostasis $[11,12]$. While oxidative stress in neurons has complex and multifaceted effects, recent reports suggest that activation of $\mathrm{c}-\mathrm{Abl}$, a non-receptor tyrosine kinase, can be stimulated by oxidative stress. And thus, may be linked to the pathogenesis of PD, Alzheimer's disease (AD) and other neurodegenerative diseases [13, 14].

$\mathrm{c}$-Abl is a tyrosine kinase known to be activated by cellular stressors, such as oxidative stress and DNA damage $[15,16]$. c-Abl also functions to regulate many fundamental cellular processes, such as cell survival, migration, and growth factor signaling $[17,18]$. While c-Abl abnormalities are linked to leukemia and other cancers, emerging studies implicate aberrant c-Abl activity in neurodegenerative disease [13]. In $\mathrm{PD}, \mathrm{c}$ - $\mathrm{Abl}$ is activated in regions showing DAergic neurodegeneration, such as the striatum and SNpc, and inactivates parkin by phosphorylation [1922]. Significantly, c-Abl activation is linked to $\alpha \mathrm{S}$ pathology as increased $\alpha \mathrm{S}$ expression in cells and Tg mice was associated with $\mathrm{c}-\mathrm{Abl}$ activation, and inhibition of $\mathrm{c}-\mathrm{Abl}$ [23] or the loss of c-Abl expression [24] leads to attenuation of $\alpha \mathrm{S}$ levels and/or aggregation. Some of these studies implicate c-Abl as an inhibitor of autophagy [25, 26]. However, it is unknown how c-Abl regulates autophagy. Alternatively, a recent study proposed that direct phosphorylation of $\alpha \mathrm{S}$ by c-Abl might be involved in the regulation of $\alpha$-synucleinopathy by c-Abl [24, 27]. However, given that c-Abl-dependent tyrosine phosphorylated $\alpha \mathrm{S}$ does form fibrils efficiently in vitro [28, 29], it is unclear that increased c-Abl activation can directly increase $\alpha \mathrm{S}$ aggregation. Finally, it is currently unknown if pharmacological inhibition of $\mathrm{c}$-Abl in adult animals can attenuate onset and progression of overt $\alpha$-synucleinopathy.

In previous studies linking $\alpha$-synucleinopathy and c-Abl activation, a key pathway traditionally associated with the cAbl activation was not addressed. Specifically, c-Abl can regulate p53 activity via phosphorylation of $\mathrm{Mdm} 2 / \mathrm{Hdm} 2$ [30], an E3 ligase responsible for degradation of p53. In the past, we showed that in the TgA53T model, neurodegeneration is associated with an increase in neuronal p53 expression [31]. Thus, we examined whether the $\alpha \mathrm{S}$ dependent c-Abl activation was associated with increased p53 activation. Herein, we show increased activation of both c-Abl and p53 with $\alpha$-synucleinopathy in a TgA53T mouse model of $\alpha$-synucleinopathy [32] and in human PD cases. Fundamentally, a significant fraction of active p53 was mislocalized to the cytosolic compartment of neurons exhibiting $\alpha \mathrm{S}$ pathology, a phenomenon shown to inhibit autophagy [33]. Indeed, we show that c-Abl-dependent inhibition of autophagy is p53 dependent. Further, both c-Abl and p53 activity is positively associated with mTOR activity and inversely associated with AMPK/ULK1 activity, showing that c-Abl and p53 directly impact the pathways relevant to autophagy regulation. Finally, we show that c-Abldependent pathway is a significant target for therapeutic intervention as pharmacological inhibition of $\mathrm{c}$-Abl delays disease onset in two independent Tg mouse models of $\alpha$ synucleinopathy. Our data identify a novel pathway for regulation of autophagy in $\alpha$-synucleinopathy and support the development of c-Abl and p53 inhibitors for disease modifying therapies for PD and other $\alpha$-synucleinopathies.

\section{Methods \\ Animals}

The generation of transgenic (Tg) mice (TgA53T) that express high levels of human mutant A53T $\alpha \mathrm{S}$ under the control of a mouse prion protein promoter have been described previously [31, 32, 34, 35]. Mice expressing the A53T $\alpha$ S develop progressive neurological dysfunction $\sim 12$ months of age, which rapidly progress to end stage paralysis within 14-21 days following initial onset of symptoms. For this study Tg mice were evaluated at early stage defined by slight instability, bradykinesia, and ataxia. End-stage was defined by the onset of paralysis in all limbs. Asymptomatic mice were 10-14 months old but did not display any motor deficits. Age matched non-transgenic (nTg) littermates were used as controls. At 9 months of age, a cohort of animals including both $\mathrm{Tg}$ and $\mathrm{nTg}$ were subjected to treatment with $10 \mathrm{mg} / \mathrm{kg}$ Nilotinib or DMSO (Vehicle) control. Animals were dosed by intraperitoneal (i.p.) injections three times weekly until animals displayed end-stage phenotypes described above. All experimental protocols involving mice were in strict adherence to the NIH Animal Care and Guidelines and were approved by the Institutional Animal Care and Use Committee at the University of Minnesota. We used about equal number of male and female mice in this study.

\section{Induction of a-synucleinopathy by inoculation}

Lysates used for all injections were generated from tissues of $\operatorname{TgA53T}$ mice at either 4 months of age (asymptomatic) 
or end-stage. Brainstem (BST) and spinal cord (SPC) were combined and processed together, as both regions demonstrate robust $\alpha S$ pathology at the end-stage [32]. The $3000 \times g$ lysate were prepared as previously described [36].

All stereotaxic injections were performed unilaterally into the right hemisphere in $\mathrm{HuA53TaS}$ mice at aged 6 months. Animals were anesthetized by Ketamine/Xylazine mixture $(100 / 10 \mathrm{mg} / \mathrm{kg}$, i.p.) and stereotaxically injected with $2.5 \mu \mathrm{g}$ of total protein in $2.5 \mu \mathrm{l}$. The injections occurred at a rate of $0.1 \mu \mathrm{l}$ per minute using a $28 \mathrm{~g}$ needle attached via tubing to a Hamilton syringe controlled by a constant pressure syringe pump (Harvard Apparatus, Holliston, MA). The stereotaxic coordinates used for the injection sites were as follows: intracortical/ intrastriatal (IC/IS): $2.0 \mathrm{~mm}$ lateral from the midline, + $0.2 \mathrm{~mm}$ relative to bregma, and 0.8 and $2.6 \mathrm{~mm}$ deep from the dura. Two weeks following the inoculation surgery, the animals were subjected to i.p. injections of either Nilotinib $(10 \mathrm{mg} / \mathrm{kg})$ or Vehicle (DMSO) three times weekly as described above. Animals were euthanized at either pre-determined time points or upon complete hind-limb paralysis by overdose of isoflurane inhalation and tissues were harvested for either biochemistry or immunohistochemistry.

\section{Tissues from human PD and control cases}

Fresh frozen brain tissues (Pons) of PD and control human cases were obtained from the Brain Resource Center (Department of Pathology, Johns Hopkins University School of Medicine). The pathological characterizations of the tissues were done as described $[37,38]$.

\section{Antibodies}

The list of primary antibodies used in this study are listed in the supplementary table (Additional file 1: Table S1).

\section{Immunoblot analysis of protein expression}

Relative protein levels of $\alpha \mathrm{S}, \mathrm{c}-\mathrm{Abl}, \mathrm{p} 53, \mathrm{Mdm} 2$ and other proteins were determined from whole tissue extracts by quantitative immunoblots analysis [32, 37] using chemiluminescence detection of horseradish peroxidase-conjugated secondary antibodies. Images were captured using the GE Imagequant LAS-4000 (GE Healthcare, Waukesha, WI) and the Imagequant software (GE) was used to determine the intensity of the immunoreactive bands.

\section{Immunoprecipitations}

Immunoprecipitations were conducted using the Crosslink Immunoprecipitation Kit (Thermo Scientific, Rockford, IL) as previously described [37, 39]. Briefly, $1 \mu \mathrm{g}$ of Mdm2 antibody was cross-linked using $2 \mathrm{mM}$ DSS to protein $G$ agarose. Bound proteins were freed from the beads by SDS-sample buffer before separation by SDSPAGE.

\section{Immunohistochemistry}

Brains and spinal cords from appropriate mice littermates were fixed in $4 \%$ paraformaldehyde (PFA) and embedded in paraffin. Sections were cut at $7 \mu \mathrm{m}$ and immunostained using an immunoperoxidase method with diaminobenzidine (DAB) as previously described [31, 40, 41] or double immunostaining followed by confocal imaging. Alex fluor (AF-647 or AF-488) conjugated secondary antibody was used. Alternatively, fixed brains were immersed in 30\% sucrose solution and prepared for frozen sliding microtome cut at $40 \mu \mathrm{m}$ followed by immunostaining of free-floating sections [41].

\section{Cell culture}

$\mathrm{BE}(2)-\mathrm{M} 17$ cells expressing human $\alpha \mathrm{S}$ under the regulation of doxycycline [39] (Colla et al., 2012), Hippocampal neuronal cell lines, and human embryonic kidney cell line (HEK-293) were used in this study. Cells were transfected with pcDNA3.1-c-Abl-PP or pcDNA3.1-c-Abl-KDM plasmid $[42,43]$ using Lipofectamine LTX. A double proline mutation in the regulatory 'linker' region between the $\mathrm{SH} 2$ and kinase domain (P242E/P249E) confers constitutive activity to c-Abl, and the kinase dead mutant (KDM) bearing the additional K290R mutation [44]. A p53 and scramble SiRNA (GS 7157, Qiagen) were done by using DharmaFECT1 transfection reagent (T-2001-02). Nilotinib and pifithrin- $\alpha$ were administered. Cells were washed with PBS and harvested in TNES buffer for protein analysis as described above [39, 40].

\section{Statistical analysis}

To test for statistical significance between treatment groups, data was analyzed by one-way or two-way analysis of variance (ANOVA) followed by a Multiple Comparison Post-hoc test (Tukey's/Dunnett's/Bonferroni's), or Student's $t$ test using GraphPad PRISM Software (7.0). All the data are expressed as means \pm S.E. Probability (p) values less than 0.05 were considered significantly different.

\section{Results \\ a-Synucleinopathy leads to c-Abl and p53 activation}

Previous studies implicate c-Abl in the pathogenesis of Parkinson's disease (PD). c-Abl can inactivate parkin [45] or directly phosphorylates $\alpha$-synuclein $(\alpha S)[24,27]$. However, because c-Abl is known to increase p53 activation via inactivation of $\mathrm{Mdm} 2 / \mathrm{Hdm} 2$, an E3 ubiquitin-ligase for p53 [30, 46], we propose that the pathogenic action of c-Abl in $\alpha$-synucleinopathy may involve p53. Thus, we determined if both c-Abl and p53 are coordinately activated in the TgA53T model of $\alpha$-synucleinopathy [32]. Progressive neurodegenerative diseases in these mice are associated with signs of oxidative stress, as indicated by mitochondrial abnormalities [31] and accumulation of 
oxidative damage, including accumulations of 3nitrotyrosine [47] (Additional file 1: Figure S1), conditions that promote both c-Abl and p53 activation.

Analysis of symptomatic TgA53T mice at early and end-stage of disease $[39,40]$ show that while the overall c$\mathrm{Abl}$ levels are not different from age matched non- $\mathrm{Tg}$ (nTg) mice, there is a significant increase in the level of cAbl phosphorylated at $\mathrm{Tyr}_{245}$, an indicator of active c-Abl, in regions affected by $\alpha$-synucleinopathy [brainstem (Fig. 1) and spinal cord (Additional file 1 : Figure S2)]. We can exclude c-Abl activation as a consequence of $\alpha \mathrm{S}$ overexpression based on the facts that: 1) c-Abl activation is absent in TgA53T cortex (Fig. 1a), which lack significant $\alpha \mathrm{S}$ pathology despite high levels of mutant $\alpha \mathrm{S}$ expression [32]; and 2) analysis of brain stem regions from asymptomatic TgA53T mice lacking $\alpha$ S pathology do not show increased c-Abl (Fig. 1a). Collectively, these data show that c-Abl activation in TgA53T mice require overt $\alpha \mathrm{S}$ pathology.

Analysis of p53 levels show that the relative levels of active p53, the phospho-Ser ${ }_{15}$ (pS15p53), mirrors the increase in the c-Abl activation (Fig. 1b, Additional file 1: Figure S2) as the abundance of active p53 is higher in the subcortical areas of symptomatic TgA53T mice (Fig. 1b; Additional file 1: Figure S2) and progressively

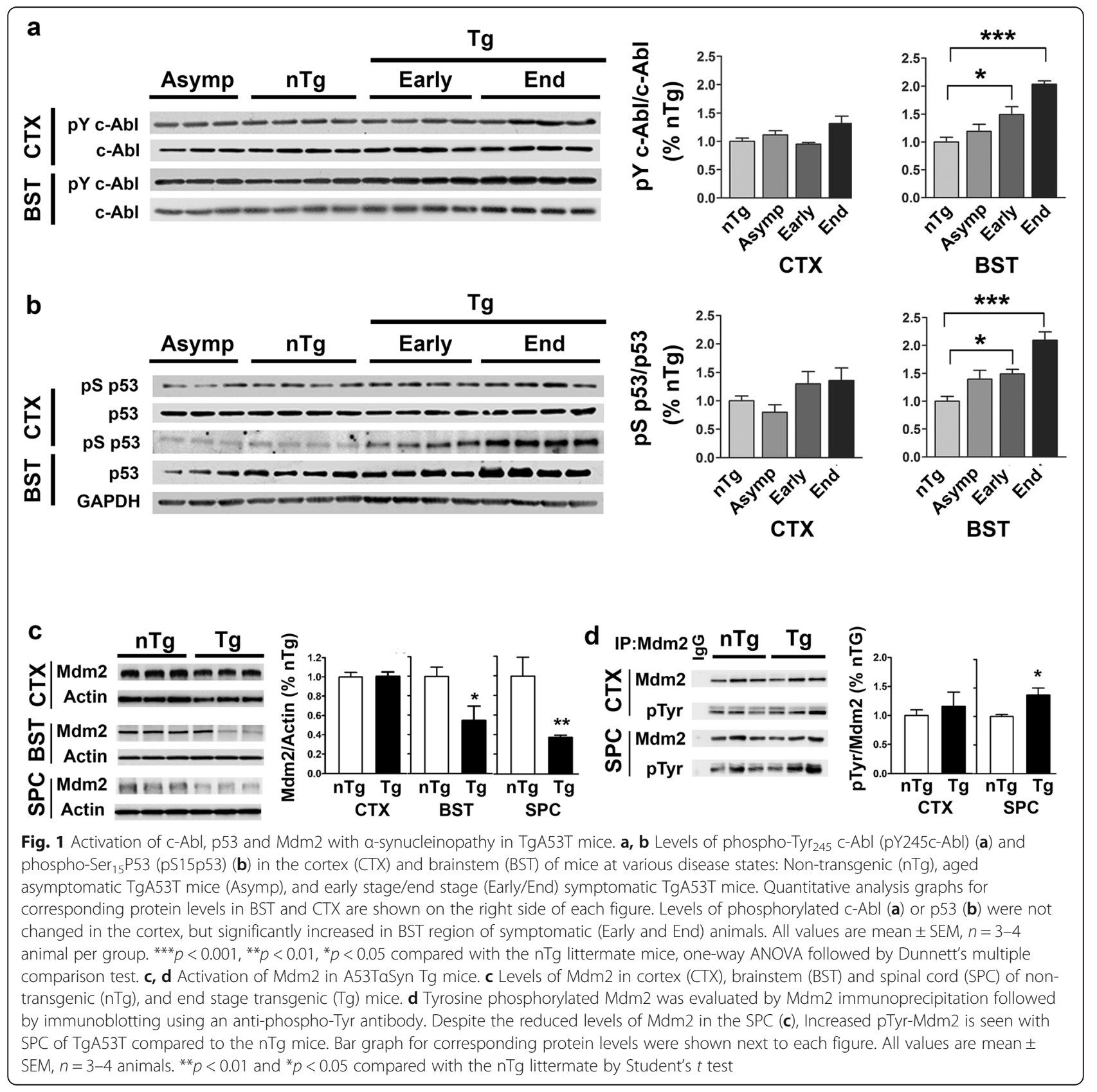


increases with the progression of disease (i.e., end-stage showing higher levels of active p53 than the early stage symptomatic TgA53T mice). As with the activation of c$\mathrm{Abl}$, activation of p53 is not due to simple overexpression of the mutant $\alpha \mathrm{S}$ as the levels of active p53 in TgA53T mice was not increased in absence of significant $\alpha$-synucleinopathy, such as in cortex and asymptomatic animals (Fig. 1b).

We then examined whether the increased activation of c-Abl and p53 was associated with abnormalities in Mdm2. Analysis of Mdm2 in end-stage TgA53T and nTg littermate mice show reduced total Mdm2 levels in both the brainstem and spinal cord regions but not in the cortex (Fig. 1c). We also examined whether the reduced Mdm2 levels in TgA53T was associated with increased tyrosine phosphorylation by immunoblot analysis of immunoprecipitated Mdm2 for phosphor-Tyr (Fig. 1d; Additional file 1: Figure S3). The results show that, consistent with $\mathrm{Mdm} 2$ being a substrate for c-Abl, the reduction in Mdm2 was associated with increased tyrosine phosphorylation of Mdm2 (Fig. 1d).

\section{Cellular localization of active c-Abl and p53 is coincident with neuronal a-synucleinopathy}

To determine if c-Abl and p53 activation occurs in neurons exhibiting $\alpha \mathrm{S}$ pathology, we performed double immunofluorescence analysis for $\alpha \mathrm{S}$ pathology (pS129 $\alpha \mathrm{S}$ ) and either pY245c-Abl or pS15p53 in brainstem from TgA53T and nTg mice. As expected, nTg mice did not show expression of pS129 $\alpha$ S, pY245c-Abl, or pS15p53 (Fig. 2a,b). In contrast, TgA53T mice show clear neuronal expression of pS129 $\alpha$ S, pY245c-Abl, and pS15p53 (Fig. 2a,b). Moreover, virtually all pY245c-Abl and pS15p53 expression was associated with neurons containing pS129 $\alpha$ S (Fig. 2a,b). These results demonstrate that the increased c-Abl and p53 selectively occurs in neurons harboring $\alpha \mathrm{S}$ pathology.

A notable aspect of the pS15p53 immunostaining in TgA53T neurons was that, instead of the usual nuclear localization of p53, pS15p53 was prominently localized to cytosolic compartment (Fig. 2b, arrow). Analysis of total p53 distribution using a pan-p53 antibody also showed an substantial increase in the neuronal p53 staining in TgA53T mice compared to nTg mice (Fig. 2c), with the increased nuclear p53 staining in TgA53T mice (Fig. 2c, arrow). Significantly, in addition to the increased nuclear p53 in TgA53T neurons, there was clear cytosolic accumulation of p53 in the TgA53T neurons (Fig. 2c, arrowhead), whereas p53 was restricted to nucleus (arrow) in nTg neurons. Analysis of p53 localization in other brain regions affected by $\alpha \mathrm{S}$ pathology also show dramatic cytosolic mislocalization of p53, where p53 accumulation extends to the proximal neurites (Additional file 1: Figure S4). These results are consistent with our hypothesis that the active pS15p53 accumulates in the cytosol because Mdm2 does not target p53 for proteasomal degradation.

\section{a-Synucleinopathy in TgA53T mice is associated with dysregulation of autophagy-lysosomal process}

The cytosolic accumulation of active p53 with $\alpha$ synucleinopathy is of interest because cytosolic p53, but not nuclear $\mathrm{p} 53$, is implicated as a negative regulator of autophagy [33]. Thus, we asked whether robust cytosolic neuronal accumulation of p53 in TgA53T mice is associated with signs of impaired autophagy. As an indicator of autophagic status in TgA53T mice, we examined the expression of p62, an adaptor molecule and recruit substrates to autophagosomes, and LC3-II, a marker of autophagosome biogenesis and interact with p62 [48]. Our results show that brain regions with $\alpha \mathrm{S}$ pathology in TgA53T mice exhibit significant increases in both p62 and LC3-II levels compared to the levels in $\mathrm{nTg}$ mice (Fig. 3a). We also examined whether abnormal autophagy, as indicated by p62 accumulation is coincident with the presence of $\alpha \mathrm{S}$ pathology. Double immunofluorescence analysis shows that the increase p62 occurs in neurons containing $\alpha \mathrm{S}$ pathology (Fig. 3b; Additional file 1: Figure S5). Normally, p62 binds with LC3-II to transport defective cargo to the autophagosome for degradation [49]. Thus, when autophagy is inhibited, particularly at the level of autophagosome fusion with the lysosomes, both p62 and LC3-II accumulates [50]. The fact that both LC3-II and p62 accumulates indicate that $\alpha$-synucleinopathy in TgA53T mice is associated with the dysfunctional autophagy, particularly at the step of autophagosome clearance.

\section{Human PD cases exhibit c-Abl activation and p62 accumulation}

To determine whether increased c-Abl and p53 activation also occurs in human $\alpha$-synucleinopathy, we examined human postmortem brain tissue from PD cases (Fig. 3c). To correlate c-Abl/p53 activation with $\alpha \mathrm{S}$ pathology, independent of ongoing dopaminergic neurodegeneration, we examined the Pontine region from human subjects as this region shows most significant $\alpha \mathrm{S}$ pathology in PD cases $[38,51]$. As in TgA53T mice, the presence of $\alpha S$ pathology is associated with significant increases in the levels of active c-Abl and total p53 in PD cases compared to age matched controls (Fig. 3c). Previously reported, increased p53 occurs in the cytosolic compartments of neurons in variety of neurodegenerative diseases, including PD, AD, DLBD and ALS [52]. Collectively, our results and previous p53 localization study [52] support the view that TgA53T model is reflecting the abnormalities seen in human $\alpha$ synucleinopathy. Consistent with this view, analysis of the pontine region also reveals a significant increase in 


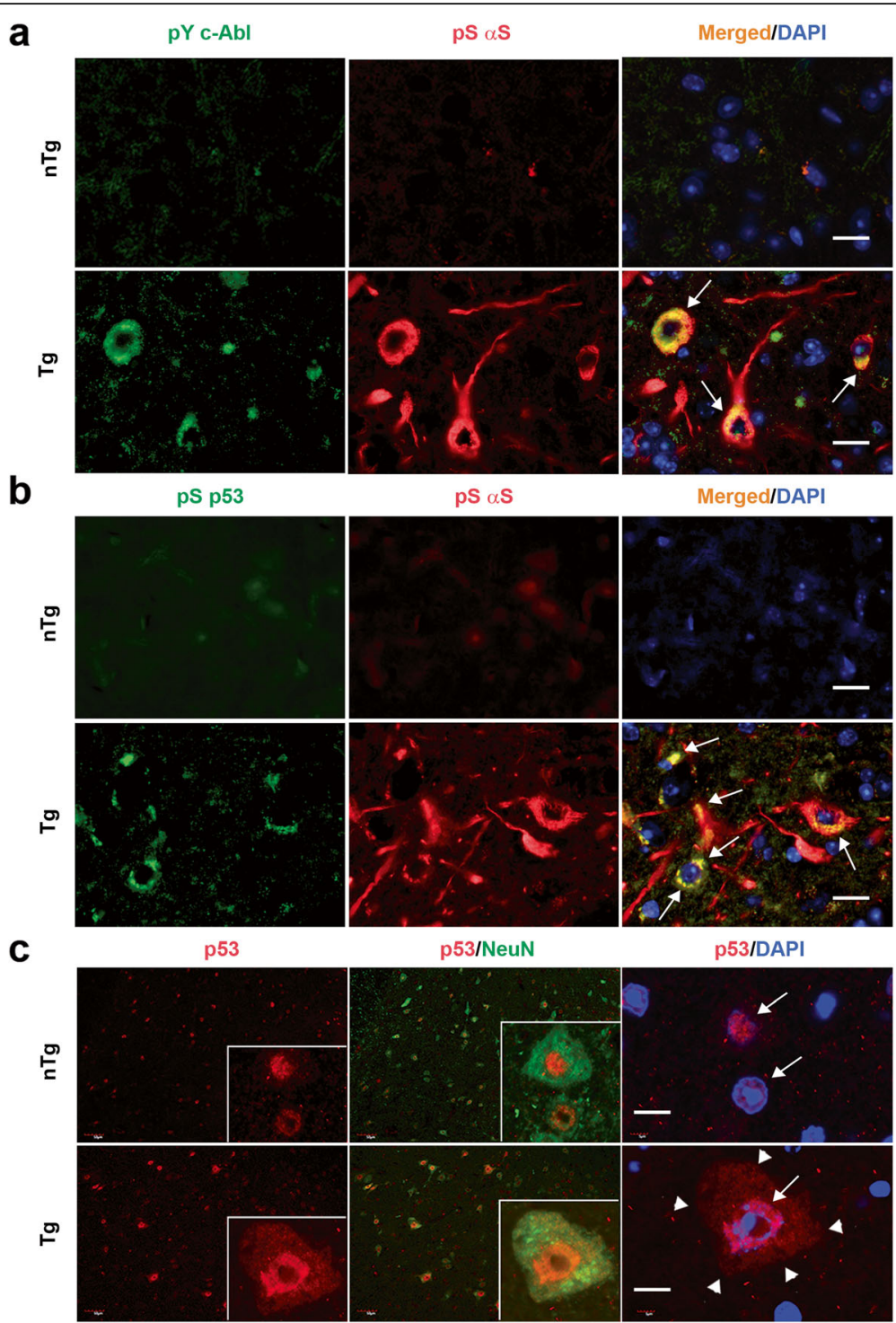

Fig. 2 Activated c-Abl and p53 accumulates in neurons exhibiting aS pathology. a, b Pathological aS accumulation in the brainstem of end-stage TgA53T Hu aS mice were detected using anti-pS129aS antibody (Red). The sections were also double immune-stained for activated c-Abl (pY245c-Abl, Green) (a) or activated p53 (p515p53, Green) (b). Arrows indicate colocalization of active c-Abl (a) and p53 (b) with neurons that accumulate pS129aS. No significant staining for pS129aS, pY245c-Abl, or pS15p53 are seen in nTg mice. Scale, 20 um. c. Spinal cord (SPC) sections from nTg and end stage TgA53T mice were immunostained using a pan-p53 antibody followed by a confocal fluorescence imaging. Note the overall increase in the p53 immunoreactivity in TgA53T mice compared to $\mathrm{nTg}$ mice. Arrows shows the nuclear localization of p53 in both $\mathrm{nTg}$ and $\mathrm{Tg}$. Arrowhead shows the cytosolic localization of p53 seen only in the TgA53T mice. Scale, $10 \mu \mathrm{m}$

the level of autophagy substrate p62 in the PD cases compared to the age matched controls (Fig. 3d). In contrast, p62 accumulation is absent in cortex of PD cases (Fig. 3e), suggesting autophagy impairment in human $\mathrm{PD}$ cases is also associated with $\alpha \mathrm{S}$ pathology.

\section{C-Abl activation causes p53-dependent autophagy defect} Thus far, our results support the hypothesis that c-Abldependent accumulation of p53 leads to autophagy defect in TgA53T mouse model. To more directly test the link between c-Abl, p53, and autophagy, we used cellular models to test if increased c-Abl activity is sufficient to inhibit autophagy in a p53-dependent manner. First, we used constitutively active c-Abl (c-Abl-PP) [42, 43] to drive c-Abl activity in HEK-293 cells and M17 neuroblastoma cells (Fig. 4; Additional file 1: Figure S6, S7, S8). In both cell types, expression of c-Abl-PP lead to significant increase in c-Abl activity, as indicated by the increased $\mathrm{Tyr}_{412}$ autophosphorylation of c-Abl (Figs. 4; Additional file 1: Figure S6, S7, S8). Increased c-Abl activity was also associated with clear deficits in the autophagy flux compared to the empty vector alone in HEK293 cells (Fig. 4a; 


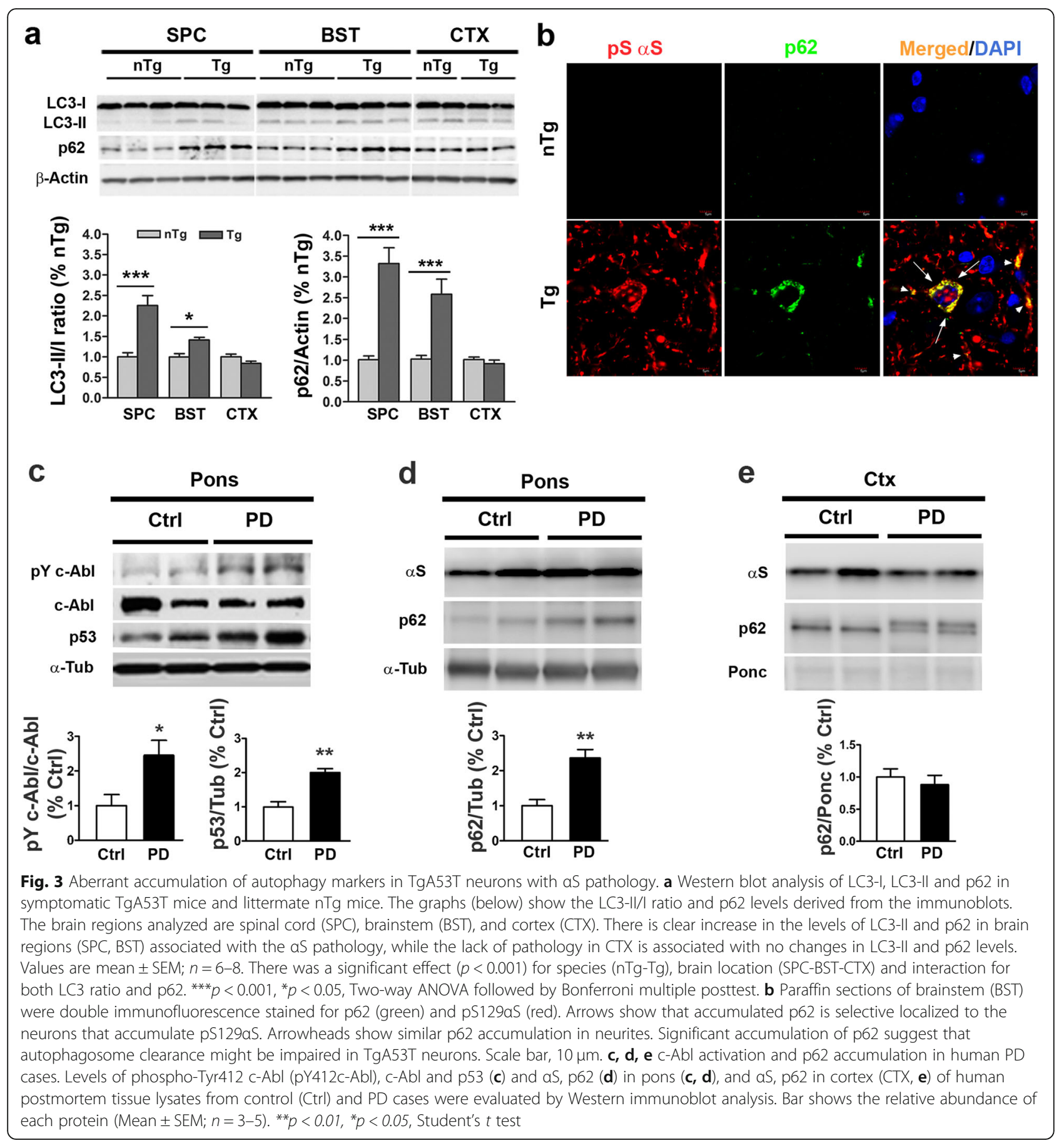

Additional file 1: Figure S6) and in M17 mouse neuroblastoma cells (Fig. 4b). In addition to basal autophagy, activation of c-Abl significantly inhibited autophagy following nutrient starvation (Additional file 1: Figure $\mathrm{S} 7 \mathrm{a})$, demonstrating that c-Abl regulates the key steps in autophagy involved in multiple types autophagy activation. Inhibition of autophagy by c-Abl requires the kinase activity as autophagy was not inhibited by the kinase-dead cAbl (KDM) [43] (Additional file 1: Figure S7b). Overall, these results demonstrate that increased c-Abl activation is sufficient to inhibit multiple types of autophagy. Because c-Abl-PP overexpression lead to defects in autophagy flux and reductions in both LC3-I and LC3-II levels, c-Abl may inhibit early stages of autophagosome formation by reducing LC3 expression.

We then examined the role of $\mathrm{p} 53$ in the c-Abl dependent inhibition of autophagy. First, expression of constitutively active c-Abl-PP expression leads to significant decrease in 

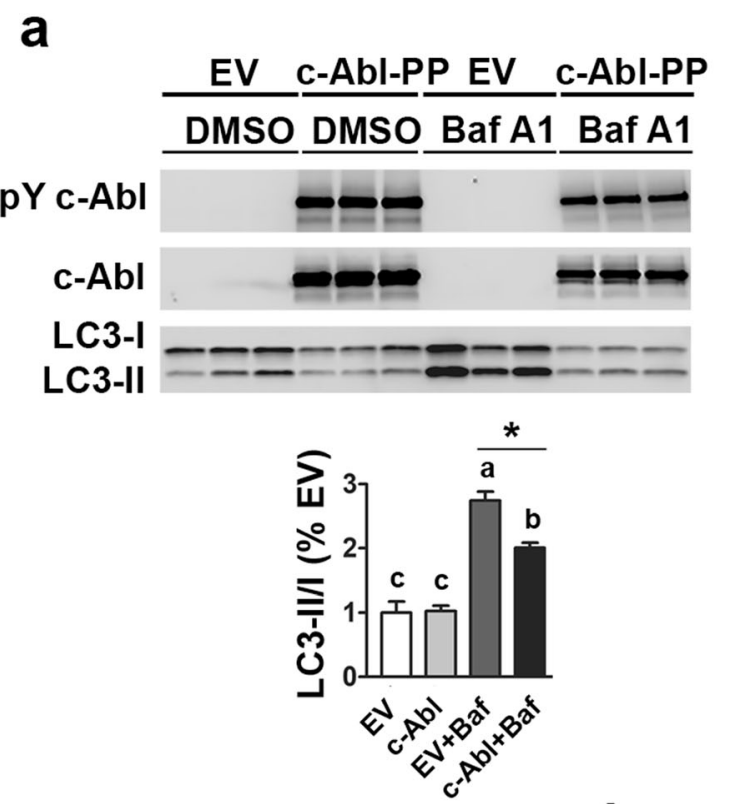

C
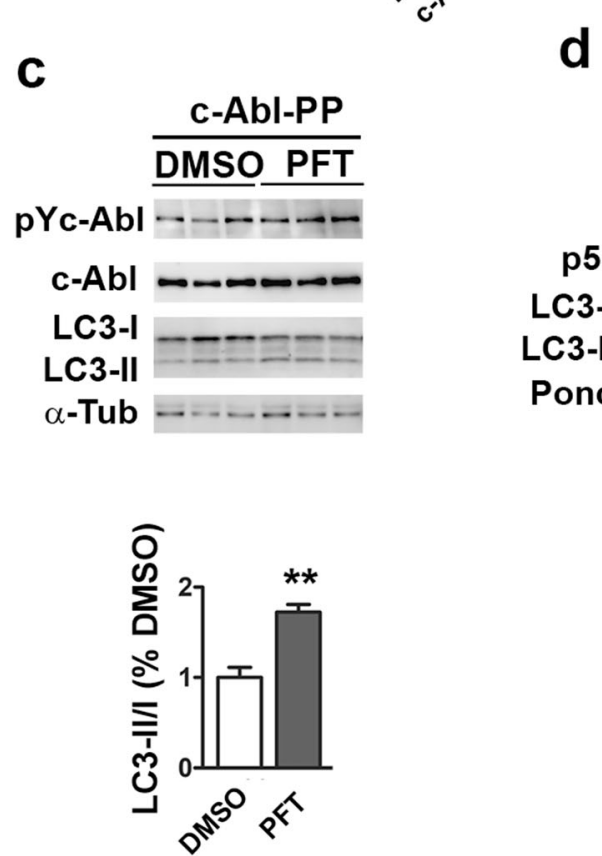

b
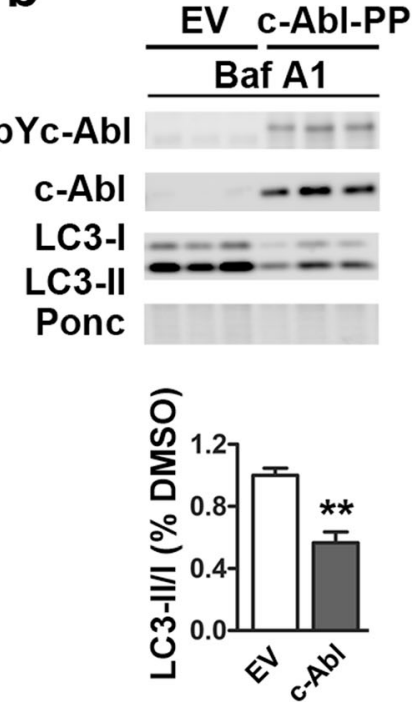
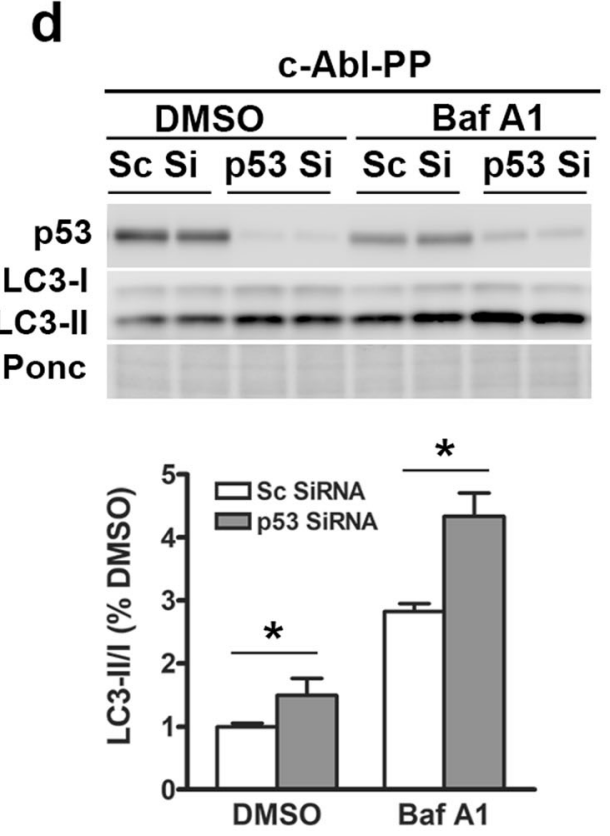

Fig. 4 Constitutively active c-Abl causes p53-dependent autophagy deficit. a, b, c Human embryonic kidney cells, HEK-293 (a, c) or M17 human neuroblastoma cells $(\mathbf{b})$ were transfected with empty vector (EV) or constitutively active c-Abl (c-Abl-PP) and, at $24 \mathrm{~h}$ post-transfection, the cells were treated with DMSO or Bafilomycin A1. Total lysates were immunoblotted for pY412c-Abl, c-Abl, and LC3. The quantitative analyses of the immunoblots show means \pm SEM $(n=3)$. The results show that the expression of c-Abl-PP leads to suppression of autophagy $(\mathbf{a}, \mathbf{b})$. $\mathbf{c}, \mathbf{d}$ HEK293 cells expressing c-Abl-PP were treated with a p53-inhibitor (pifithrin-a; PFT) (c) or p53 siRNA (Si) (d). In (d) scrambled siRNA (Sc Si) were used as a control and cells were further treated with DMSO or Bafilomycin A1. Analysis of pY412c-Abl, c-Abl, p53 and LC3 show that both pharmacological (PFT, $\mathbf{c}$ ) or genetic (p53 Si, d) inhibition of p53 reverses the effects of c-Abl-PP. a a ${ }^{a, b}$ Significantly different from $c, p<0.01$, ${ }^{*} p<0.05$, One-way ANOVA with Tukey's multiple comparison posttest. b, $\mathbf{c}, \mathbf{d}^{*} p<0.05 ;{ }^{* *} p<0.01$, Student's $t$ test

Mdm-2 levels and increased p53 levels (Additional file 1: Figure S8). More important, active p53 is required for c-Abl dependent inhibition of autophagy, as inhibition of p53 by pifithrin- $\alpha$ (PFT) was able to block autophagy defect in cAbl-PP expressing cells (Fig. 4c). To further demonstrate that p53 mediates autophagy inhibition by c-Abl, we used
siRNA to knockdown p53 (Fig. 4d). Compared to the scrambled siRNA, p53 siRNA was able achieve $>80 \%$ reduction in p53 levels. Analysis of LC3-I and LC3-II show that as with PFT treated cells, p53 knockdown was able to rescue defects in autophagy flux in c-Abl-PP transfected cells (Fig. 4D). 
Upon establishing that c-Abl activation inhibits autophagy in a p53 dependent manner, we asked whether c-Abl and p53 could be targeted to enhance basal autophagy in neuronal cells. As expected, a c-Abl inhibitor, nilotinib (Nilo), induces basal autophagy flux, as indicated by the analysis of bafilomycin A1 (BafA1) dependent the turnover of LC3-II (Fig. 5a). Our results with neuronal cells (CLU and M17 neuroblastoma cells), show that c-Abl inhibition by Nilo treatment leads to significant increase in the basal LC3-II concomitantly deceasing p62 levels compared to control cells (Fig. 5a, b). With the BafA1 treatment (Fig. 5a), there was an expected increase in the LC3-II levels with the greater BafA1-dependent increase in LC3-II with Nilo treatment. This result confirms that Nilo increases autophagy flux. Analysis of autophagy flux at multiple time points following Nilo treatment shows increased autophagy flux at $12 \mathrm{~h}$, but not at $6 \mathrm{~h}$, of Nilo treatment in CLU cells (Additional file 1: Figure S11). Analysis of c-Abl showed that the Nilo was able to decrease the levels of pY412c$\mathrm{Abl}$ by $6 \mathrm{~h}$ of treatment (Additional file 1: Figure S12). Thus, inhibition of $\mathrm{c}-\mathrm{Abl}$ is followed by increased autophagy.

Further, inhibition of p53 by PFT treatment also enhanced autophagy in both CLU and M17 neuronal cell lines (Fig. 5a,b). Autophagy is implicated in the turnover of $\alpha \mathrm{S}$, particularly $\mathrm{pS} 129 \alpha \mathrm{S}[53,54]$. Thus, we examined Nilo treatment can facilitate the clearance of pathological $\alpha \mathrm{S}(\mathrm{pS} 129 \alpha \mathrm{S})$ in M17 neuroblastoma cells expressing A53T mutant human $\alpha \mathrm{S}$ (Fig. 5c). Our results show that Nilo treatment leads to obvious decrease in the $\mathrm{pS} 129 \alpha \mathrm{S}$ levels while only a modest decrease is seen in the total $\alpha \mathrm{S}$ levels (Fig. 5c). Collectively, these results support that inhibition of either c-Abl or p53 can enhance basal autophagy and promote clearance of $\alpha \mathrm{S}$ in neuronal cells.

\section{C-Abl and p53 regulate $\mathrm{mTOR}$, ULK1, and AMPK}

To understand the signaling mechanism linking autophagy to $\mathrm{c}-\mathrm{Abl}$ and p53, we explored whether $\mathrm{c}-\mathrm{Abl}$ and p53 affect cellular pathways that are known to regulate autophagy. We first examined mTOR signaling as autophagy is negatively regulated by mTOR activation. Analysis of cells expressing c-Abl-PP show that inhibition of autophagy by c-Abl-PP is associated with increased mTOR activity as indicated by increase in phospho-S6 (pS6), a marker of mTOR-dependent activation of ribosomal S6 kinase (Additional file 1: Figure S9). The c-Abl-PP-dependent increases in the levels of pS6 and phosphorylated 4EBP1 (p4EBP1), another major target of mTOR, were significantly attenuated by inhibition of p53, confirming that p53 is required for mTOR activation by c-Abl-PP (Additional file 1: Figure S10).
We also examined whether increase in basal autophagy induced by the inhibition of $\mathrm{c}-\mathrm{Abl}$ or p53 in cultured neuronal cells is associated with a decrease in mTOR activity. Inhibition of c-Abl or p53 in M17 neuroblastoma cells leads to significant decreases in the levels of pS6 and p4EBP1 (Fig. 5d,e), confirming that the autophagy induced by inhibition of c-Abl or p53 involves reduced mTOR activity. UNC-51 like kinase 1 (ULK1) and adenosine monophosphate-activated kinase (AMPK) are well known upstream regulator of mTOR activity where AMPK1 dependent activation of ULK1 is known to cause mTOR inhibition [55]. Thus, we examined the possibility that $\mathrm{c}-\mathrm{Abl} / \mathrm{p} 53$ regulates mTOR activity by effects on AMPK and ULK1. We found that the inhibition of c-Abl or p53 increases the activating autophosphorylation of AMPK (Fig. 5f,g) and active AMPK in turns facilitates autophagy by phosphorylating ULK1 at $\operatorname{Ser}_{555}$ (Fig. 5f,g), leading to ULK1 activation, and by inhibition of mTORC1 (Fig. 5d,e), which inhibits autophagy by ULK1 inactivation [56-58]. Moreover, analysis of the CLU cells at various times following Nilo treatment show that prior to autophagy activation, inhibition of c$\mathrm{Abl}$ at $6 \mathrm{~h}$ of Nilo treatment is coincident with the decrease in pS6, indicating that c-Abl inhibition us coincident with the inhibition of mTORC1 signaling (Additional file 1: Figure S12). As with the LC-3 (Additional file 1: Figure S11), down stream effector of of mTOR inhibition, activation of AMPK, seen at $12 \mathrm{~h}$, but not $6 \mathrm{~h}$, of Nilo treatment (Additional file 1: Figure S12). In summary, these data show that Nilo enhances autophagylysosomal clearance of $\alpha \mathrm{S}$ by modulating c-Abl/p53 pathway through AMPK activation and down regulation of mTORC1 signal pathway.

\section{Nilotinib treatment increases lifespan of $\mathrm{Tg}$ animals, decreases c-Abl and p53 activation, and improves autophagy}

Above studies are consistent with the hypothesis that $\alpha$ synucleinopathy dependent activation of c-Abl contributes to autophagy defect seen in TgA53T mice. Further, our results are consistent with the prior studies showing that the increased $\mathrm{c}$-Abl activity promotes $\alpha$-synucleinopathy and the loss of $\mathrm{c}$-Abl expression delays onset of $\alpha$ synucleinopathy in TgA53T mice [24]. However, because the prior study uses genetic models with life-long alterations in $\mathrm{c}-\mathrm{Abl}$ expression, translational relevance of the study could be limited. Thus, we tested whether preclinical pharmacological inhibition of c-Abl with Nilo in mature TgA53T model can attenuate the onset of pathology. We used two animal models of $\alpha$-synucleinopathy to determine if c-Abl inhibition using Nilo, an FDA approved, brain penetrant $\mathrm{c}$-Abl inhibitor [21], can attenuate $\alpha$ synucleinopathy in vivo (Fig. 6). In the first model, cohorts of TgA53T mice were allowed to age and develop the 


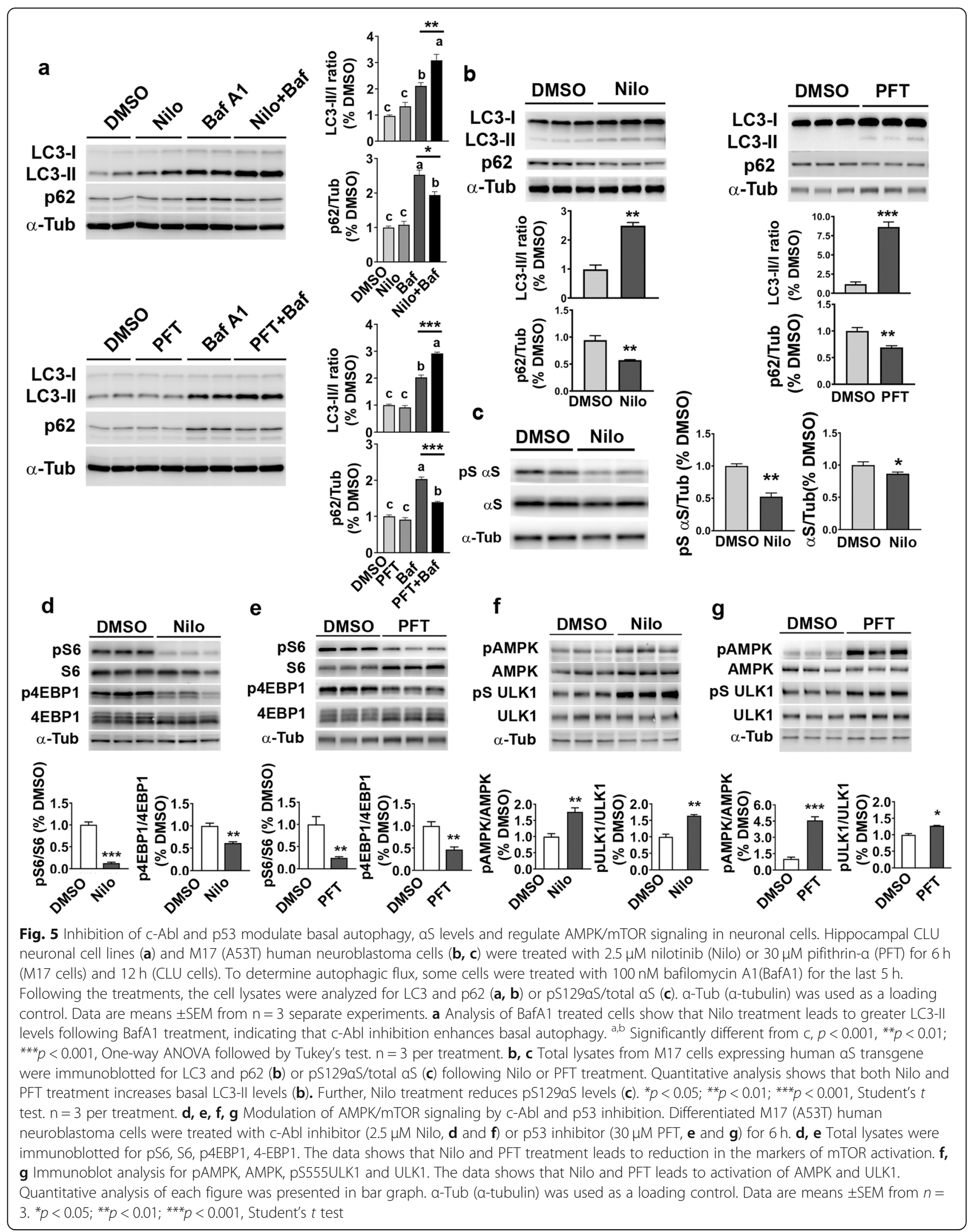




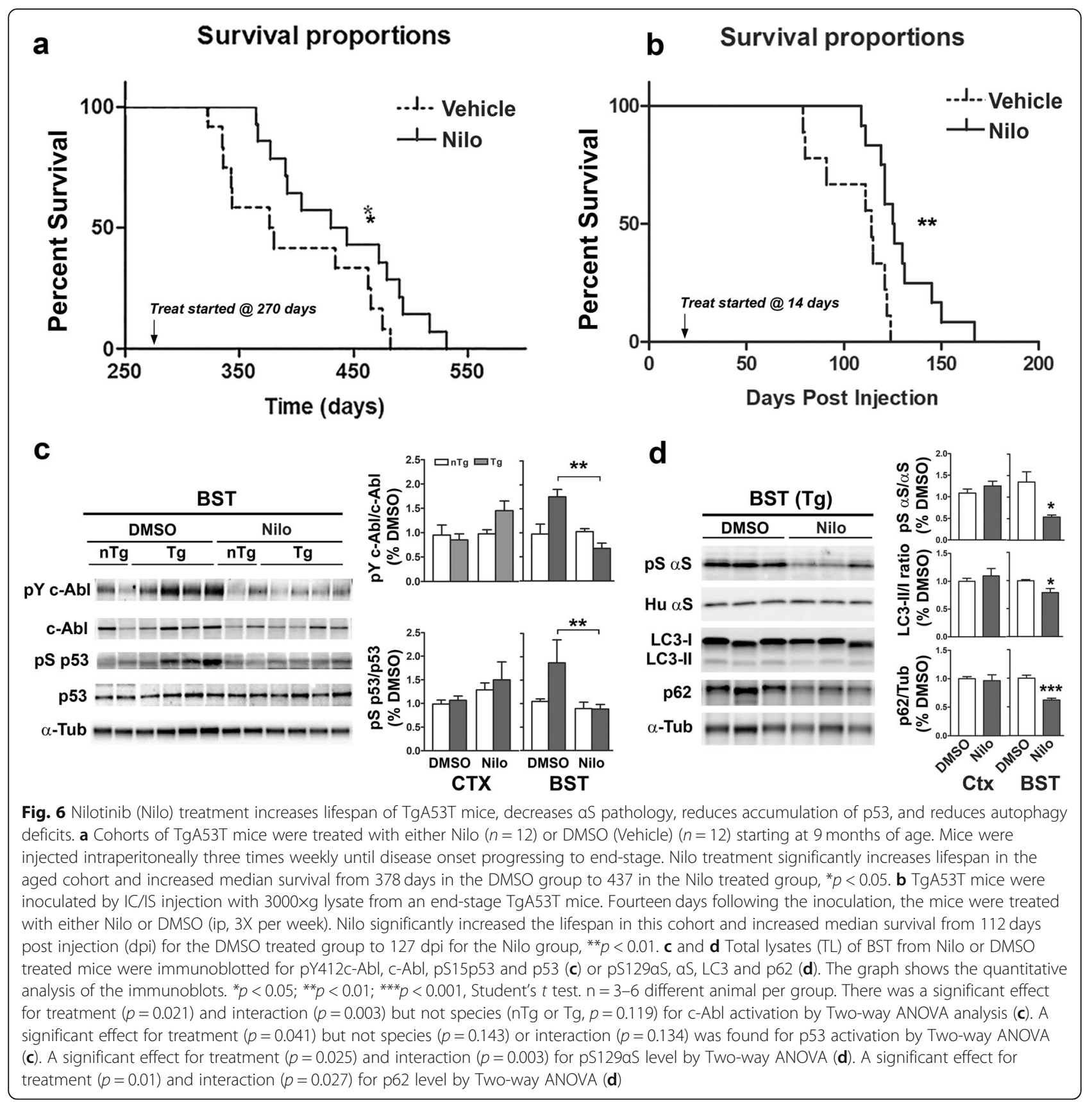

disease with aging. The Nilo/Vehicle treatments were started at $\sim 270$ days of age, when approximately $20 \%$ of the TgA53T littermates have started to show motor abnormalities, to better approximate therapeutic regimen that might be used in clinics [39] (Fig. 6a). In this model, Nilo treatment significantly increased mean average life span of the TgA53T animals compared to the vehicle treated group, from 378 days to 437 days.

The second model is established by inoculation of TgA53T mice [36] where the pathogenic lysate from end-stage TgA53T mice was injected into the cortex and striatum of pre-symptomatic Tg mice at 6 months of age
(Fig. 6b). As expected, inoculation of pathogenic lysates leads to rapid onset of motoric abnormalities between 80 and 100 days post inoculation (dpi) that progress to end-stage within 14-21 days after onset of motoric symptoms (Fig. 6b). In this model, Nilo/Vehicle treatment was initiated at 14 days post injection (dpi). As in the naturally aging model, Nilo significantly increased the mean survival from $112 \mathrm{dpi}$ to $127 \mathrm{dpi}$ in the inoculation model (Fig. 6b).

Biochemical analysis shows that, with the increase in lifespan, Nilo treatment reduced c-Abl activation in brain stem (Fig. 6c). Significantly, Nilo treatment also 
reduced the levels of active $\mathrm{p} 53\left(\mathrm{pS}_{15} \mathrm{p} 53\right)$ (Fig. 6c), confirming that in $\alpha$-synucleinopathy, accumulation of active p53 is tightly linked to c-Abl activation. No change in c-Abl or p53 activity was observed in the cortex (Fig. 6c). Furthermore, the levels of pS129 $\alpha$ S in the brainstem was significantly decreased in Nilo treated subjects compared the vehicle (DMSO) treated subjects (Fig. 6d), indicating that preclinical inhibition of $\mathrm{c}-\mathrm{Abl}$ was sufficient to decrease accumulation of $\alpha \mathrm{S}$ aggregates. In conjunction with the decrease in pathologic $\alpha \mathrm{S}$, Nilo treatment also reduced the levels of LC3-II and p62 (Fig. 6d), consistent with the idea that c-Abl inhibition is improves autophagic flux.

To further confirm that the therapeutic effects of Nilo in brainstem is linked to mechanistic events outlined using cultured cells, we compared the co-localization of p62, pY245c-Abl and pS15p53 with pS129 $\alpha$ in the brainstem neurons of DMSO (vehicle) and Nilo treated animals (Fig. 7). Both p62 and pS129 $\alpha$ S abundantly coaccumulate in TgA53T mice neurons treated with DMSO, whereas Nilo treated animal showed a comparatively lower intensity of p62 and pS129 $\alpha$ S immunoreactivity (Fig. 7a). Further, p62 in the Nilo treated neurons appear as well-defined punctate structures (Fig. 7a, arrows), suggesting that the p62 inclusions might be cargos inside autophagosome targeted for autophagic clearance. Similarly, Nilo treatment lead to overall reducing in the c-Abl and p53 immunoreactivity associated with $\mathrm{pS} 129 \alpha \mathrm{S}$ positive neurons (Fig. 7b,c). Overall, our data supports the hypothesis that c-Abl inhibition by Nilo promotes clearance of toxic $\alpha S$ via activation of autophagy.

Above results clearly indicate that Nilo treatment activates autophagy, reduces $\alpha S$ pathology, and attenuates neurodegeneration. To provide independent determinations that Nilo treatment was associated with reduced neurodegeneration, we examined the activation of astrocytes and microglia, two common indicators of neurodegeneration (Fig. 8; Additional file 1: Figure S13). Immunocytochemical analysis for astrocytes using antiGFAP antibody (Fig. 8a,b; Additional file 1: Figure S13a) and microglia using anti-IbaI antibody (Fig. 8c,d; Additional file 1: Figure S13b) shows that obvious increase in GFAP and IbaI immunoreactivity in TgA53T mice compared to nTg littermates. Significantly, Nilo treatment of TgA53T mice leads to attenuation of both GFAP and IbaI immunoreactivity (Fig. 8; Additional file 1: Figure S13), confirming that Nilo treatment attenuates neurodegeneration.

While Nilo treatment leads to dramatic reduction in $\alpha$-synucleinopathy-related abnormalities in brainstem, analysis of spinal cord reveals that Nilo was not able to reduce the levels of active c-Abl or reduce $\alpha \mathrm{S}$ pathology, including accumulation of $\mathrm{pS} 129 \alpha \mathrm{S}$, in spinal cord
(Additional file 1: Figures S14 and S15). Further, Nilo treatment did not alter autophagy markers, LC3-II and p62, in the spinal cord of TgA53T mice (Additional file 1: Figure S15). Finally, Nilo treatment did not reduce activation of astrocytes and microglia in TgA53T spinal cord (Additional file 1: Figure S16). The lack of Nilo to inhibit c-Abl and provide neuroprotection in spinal cord accounts for the modest therapeutic effect of Nilo, where the disease onset is delayed and not prevented. Significantly, the therapeutic effects seen with Nilo treatments are comparable to that achieved with the constitutive brain c-Abl-knockout mice [24]. Because, only the brain stem was analyzed for pathology in the c-Abl-knockout study [24], the status of neuropathology in the spinal cord is unknown. Regardless, in addition to the resistance of spinal cord c-Abl to inhibition by Nilo, it is possible that c-Abl-independent processes, such as chronic endoplasmic reticulum stress $[39,40]$, contribute to neurodegeneration in spinal cord neurons.

We also examined whether Nilo treatment was delaying the progression of $\alpha$-synucleinopathy by enhancing autophagy during the presymptomatic stage of the disease. For this study, we evaluated the autophagy related parameters at $60 \mathrm{dpi}$ of toxic lysates inoculated into IC/IS, well prior to disease onset at $\sim 100 \mathrm{dpi}$. The cohorts of mice were treated with DMSO or Nilo from 14 to $60 \mathrm{dpi}$ (Fig. 9a). At this time point, our analysis shows that $\alpha \mathrm{S}$ pathology is starting to appear (data not shown), but the mice have yet to develop any clinical symptoms. Our analysis shows that even at this early stage, both pS129 $\alpha$ S and total $\alpha \mathrm{S}$ levels were significantly decrease by Nilo treatment (Fig. 9b,c). Consistent with the improved autophagic flux (Fig. 6), Nilo significantly decreased LC3-II and p62 levels at the presymptomatic stages (Fig. 9b, d). We also found that Nilo leads to inhibition of mTORC1, indicated by significant reductions in the levels of S6 and 4EBP1 phosphorylation (Fig. 9b,e). Collectively, our data indicate that Nilo can induce autophagy via mTOR inhibition at early stages of $\alpha$-synucleinopathy.

\section{Discussion}

Current study investigated the pathological connection between non-receptor tyrosine kinase c-Abl and $\alpha$ synucleinopathy. Increased c-Abl activity has been found in many neurodegenerative diseases and in PD, c-Abl activation in sporadic PD has implicated parkin, an E3 ubiquitin ligase associated with autosomal recessive $\mathrm{PD}$, as potential pathogenic target $[13,19]$. However, recent studies indicate additional pathogenic target of c-Abl. For example, cAbl has been shown to phosphorylate $\alpha \mathrm{S}$ [27], potentially leading to increase $\alpha \mathrm{S}$ aggregation [24] and cAbl inhibition promotes $\alpha S$ clearance via activation of autophagy [23]. This study investigates an alternate pathway where $\mathrm{c}-\mathrm{Abl}$ activation inactivates $\mathrm{Mdm} 2$, leading to the 


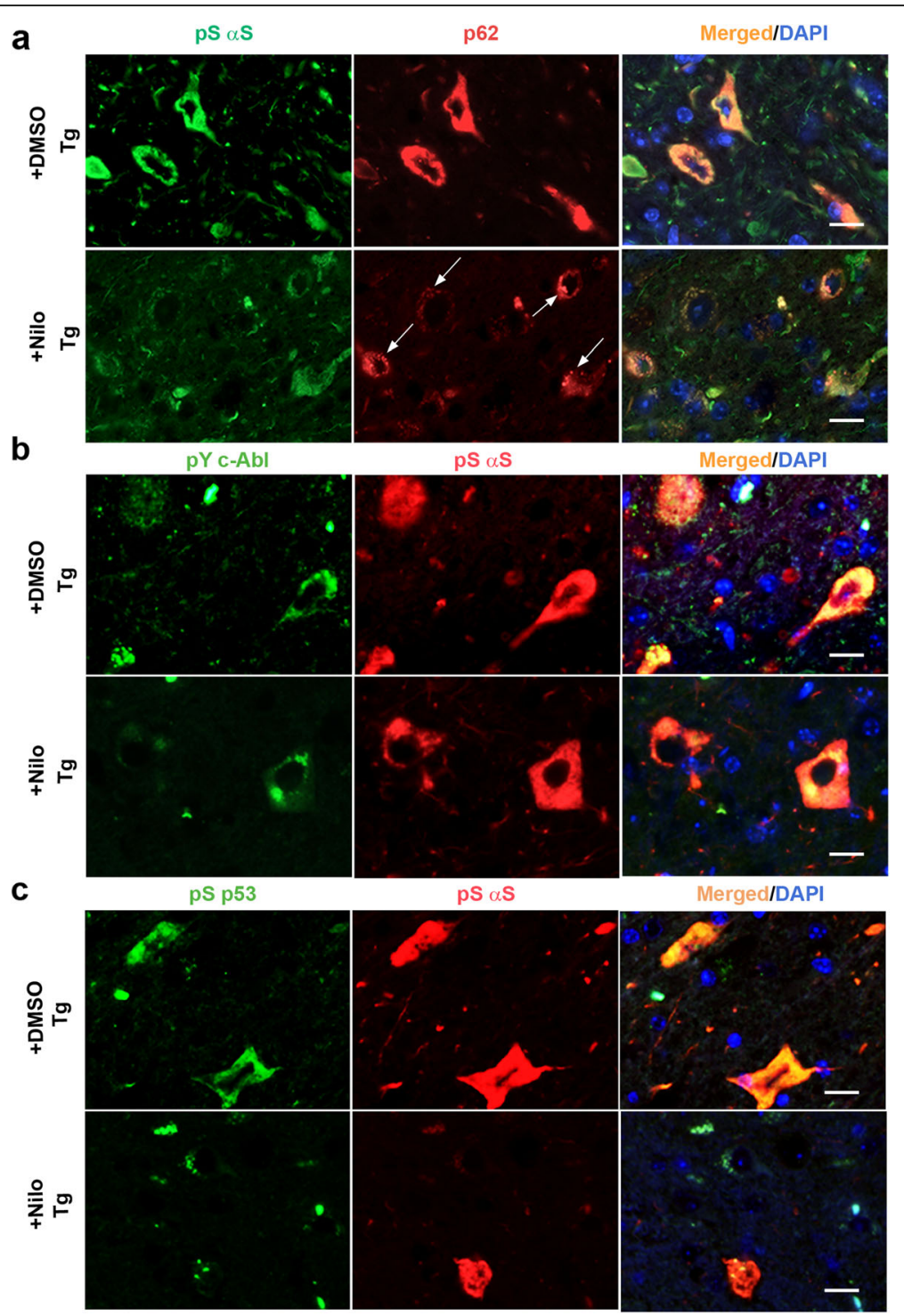

Fig. 7 Nilotinib (Nilo) treatment reduces neuronal accumulation of pS129aS, activated c-Abl, activated p53, and p62. Paraffin embedded sagittal brain sections from Nilo and DMSO treated end-stage TgA53T mice were used for immunofluorescence co-localization of pS129aS with p62 (a), pY245c-Abl (b) and pS15p53 (c). Shown are the brainstem regions. The results show that Nilo treatment leads to reduced neuronal accumulation of pS129as (a-c), p62 (a), pY245c-Abl (b), and pS15p53 (c). Scale bar, $20 \mu \mathrm{m}$

accumulation of p53 in the cytoplasm and impaired autophagy. We show that activation of c-Abl in $\alpha-$ synucleinopathy is associated with increased activation of p53. We also show that c-Abl-dependent inhibition of autophagy requires p53 activity and the c-Abl/p53 activity is associated with mTOR signaling. Further, inhibition of cAbl kinase by nilotinib (Nilo), an FDA approved inhibitor of c-Abl, is coined with increasing AMPK/ULK1 activity to enhance autophagy clearance of pathologic $\alpha$ S. Finally, we show that Nilo treatment of TgA53T mouse model delays onset of clinical symptoms and facilitate autophagic clearance of pathological $\alpha S$. Overall, our results support the novel conclusion that $\alpha$-synucleinopathy-dependent activation of $\mathrm{c}-\mathrm{Abl} / \mathrm{p} 53$ is a key underlying cause of autophagy deficit in $\alpha$-synucleinopathy and that c-Abl/p53 activity is a significant target for therapeutic intervention in $\alpha$-synucleinopathies.

\section{C-Abl/Mdm2/p53 pathway and its implication in a- synucleinopathy}

Many studies have established the c-Abl/Mdm2/p53 pathway $[16,59-62]$. c-Abl is known to interact with both Mdm2 and p53 [16, 60]. Mdm2 antagonizes p53 by ubiquitinating p53 and targeting p53 for the proteasomal degradation, as well as binding to the $\mathrm{N}$-terminus of $\mathrm{p} 53$ to prevent its transcriptional activity [60]. Stabilization 


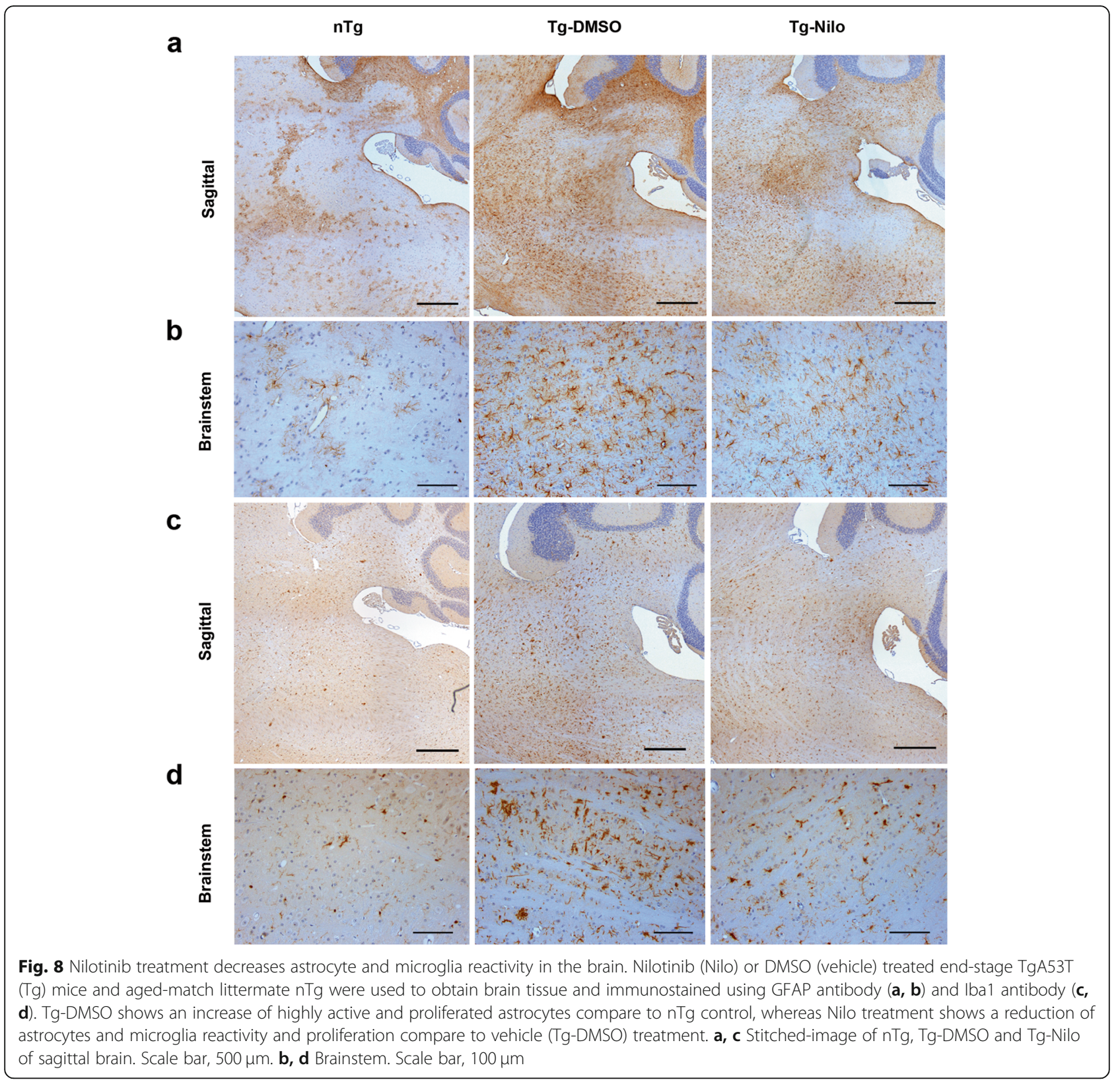

of p53 in response to cellular stress is due to a decreased Mdm2 levels and/or interactions between p53 and Mdm2 [46, 63]. Active c-Abl is known to interact with $\mathrm{Mdm} 2$ and tyrosine-phosphorylation of $\mathrm{Mdm} 2$ by c-Abl decreases its ability to degrade p53 [30,60]. We extend these studies in cancer cells to show that in neuronal cells and in brain, $\alpha \mathrm{S}$ pathology is associated with activation of c-Abl, decrease in $\mathrm{Mdm} 2 / \mathrm{Hdm} 2$ levels, and increased p53 activity.

\section{Activation of p53 involved in PD pathology and cytosolic p53 impairs autophagic clearance}

Many studies on neurodegenerative diseases, including PD, show an increase in p53 activation [64]. Expression of p53 is increased in the diseased tissue of PD patients is also elevated in the substantia nigra neurons [52, 65, 66]. Neuronal populations undergoing apoptosis with abnormal cytosolic accumulation of p53 were previously found in this $\mathrm{A} 53 \mathrm{~T} \alpha \mathrm{S} \mathrm{Tg}$ mouse model [31]. In this study, we show that the accumulation of active c-Abl and cytosolic p53 selectively occurs in neurons with $\alpha \mathrm{S}$ pathology, supporting that $\alpha \mathrm{S}$ pathology induces c-Abl activation and subsequent activation of p53. While p53 activation is normally associated with increased autophagy, via transcriptional induction of autophagy genes [67], Tasdemir et al. showed that the accumulation of cytosolic p53, but not nuclear p53, impairs autophagy $[33,68]$. Recent study also shows that inhibition of p53 


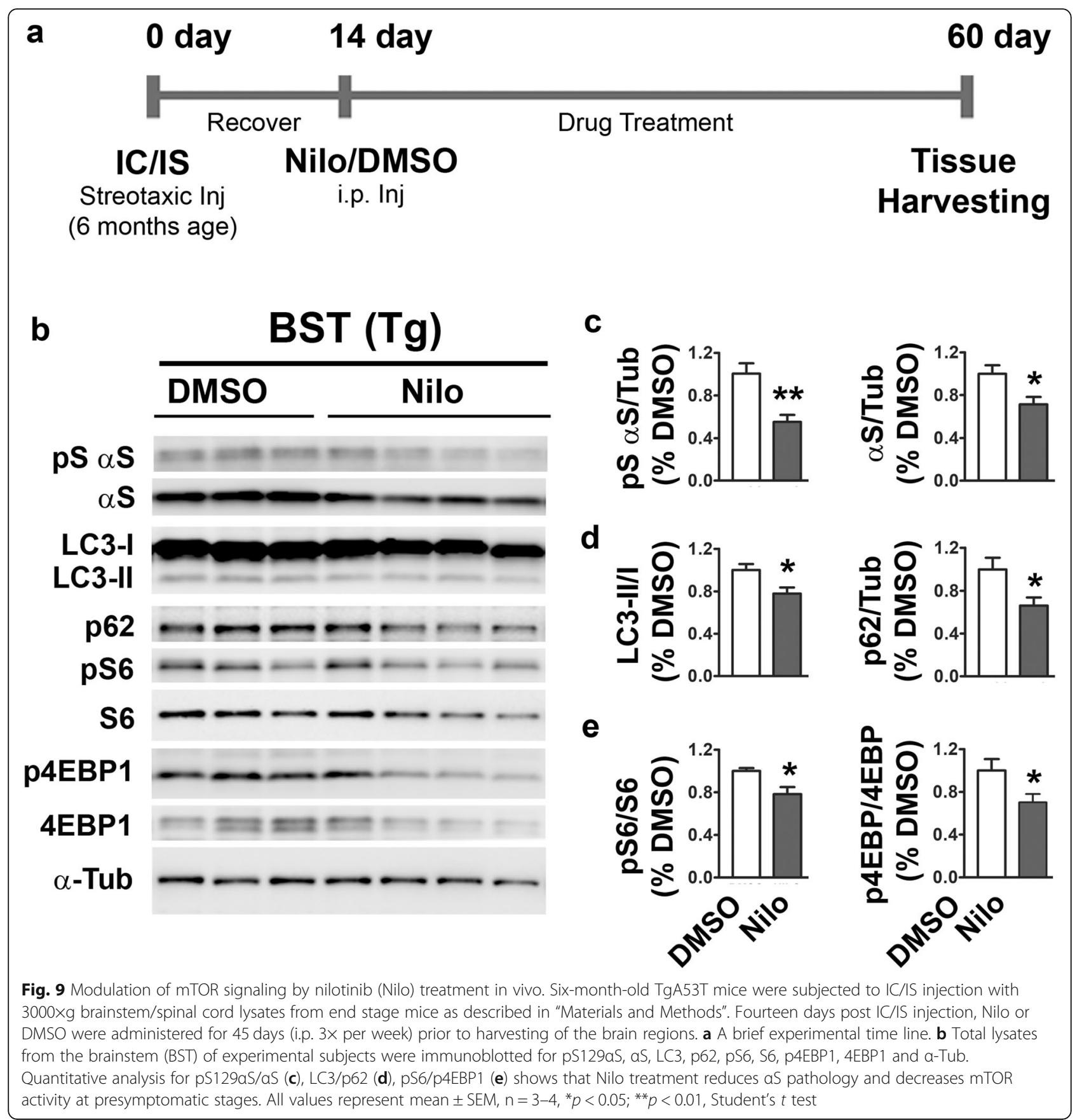

promotes parkin-mediated mitophagy in pancreatic beta cells [69]. Thus, our results are consistent with these previous studies and provide mechanistic basis for how c-Abl activation leads to autophagy deficits in $\alpha$ synucleinopathies. In this regard, studies have shown that mitochondrial toxicity is inhibited by pifithrin- $\alpha$ independent of p53 [70], indicating that pifithrin- $\alpha$ has other cellular targets. However, our results with pifithrin- $\alpha$ is likely to be p53 dependent as the p53 gene silencing was sufficient to reverse autophagy deficit caused by constitutively active c-Abl (Figs. 4 and 5).
In addition to the c-Abl-dependent p53 activation and autophagy deficits, a series of studies implicate c-Abl as a direct regulator of $\alpha$ S. Mahul-Mellier et al., (2014) showed that c-Abl directly interacts with $\alpha \mathrm{S}$ and catalyze $\alpha \mathrm{S}$ phosphorylation, mainly at tyrosine 39 (Y39) and, to lesser extent, at tyrosine 125 (Y125) [27]. A recent study [24] shows that constitutively increasing c-Abl activity in brain promotes $\alpha \mathrm{S}$ pathology and the constitutive loss of c-Abl expression in brain attenuates $\alpha \mathrm{S}$ pathology. This study implicates c-Abl in increasing $\alpha \mathrm{S}$ aggregation by $\alpha \mathrm{S}$ phosphorylation at Y39 (pY39 $\alpha$ S) [24]. However, the role of 
pY39 $\alpha$ S in promoting $\alpha \mathrm{S}$ aggregation can be debated as biochemical and biophysical studies show that pY39 $\alpha \mathrm{S}$, while more toxic to cells, aggregated much more slowly than the wild type of $\alpha \mathrm{S}$ [29]. Therefore, it is unclear that increase in pY39 $\alpha$ S level is sufficient to cause increased $\alpha S$ aggregation in cells. Rather, it is more likely that c-Abl dependent autophagy deficit, leading to accumulation of $\alpha \mathrm{S}$, is a more likely contributor to the increased $\alpha \mathrm{S}$ pathology observed in neurons with increased c-Abl activity. Thus, our hypothesis would be able to incorporate increased $\alpha \mathrm{S}$ aggregation as well as increase in the levels of toxic soluble oligomers from $\mathrm{pY} 39 \alpha \mathrm{S}$.

\section{Pharmacological inhibition of c-Abl using Nilotinib modulates autophagy and attenuates a-synucleinopathy in TgA53T model}

Studies indicate that c-Abl might be a promising therapeutic target for $\alpha$-synucleinopathy $[24,25,45,71]$ and a recent study demonstrated that a pharmacological c-Abl inhibitor, nilotinib (Nilo) enable to cross blood brain barrier [72, 73]. The previous c-Abl inhibition study [25] showed that Nilo treatment of A53T transgenic mice starting at early ages (68 months old) lead to reduced levels of total $\alpha \mathrm{S}$ levels in the forebrain regions but the effects of Nilo on overt $\alpha \mathrm{S}$ pathology $(\mathrm{pS} 129 \alpha \mathrm{S})$ and the disease onset was not reported. While constitutive loss of c-Abl expression in brain can increase the life span in TgA53T model [24], it is unclear if the attenuation of c-Abl activity at older ages, corresponding to preclinical stages of disease, has therapeutic benefit. In the current study, we show that pharmacological inhibition of c-Abl by Nilo in aged, preclinical stage TgA53T mice leads to modest but significant delay in disease onset. Further, we also show that Nilo treatment following inoculation of toxic lysate also delays disease onset, supporting the notion that c-Abl inhibition is able to slow disease initiation and disease progression. Further, we show using cell culture studies as well as biochemical analysis of mouse tissues that c-Abl activation inhibits autophagy in a p53-activity dependent manner. We also show that inhibition of c-Abl by Nilo decreases p53 levels and increase autophagy. More important, we show that Nilo increases autophagy in vitro and in vivo by activating AMPK phosphorylation and decreasing mTOR activation. Our previous study also shows that salubrinal, an anti-endoplasmic reticulum (ER) stress compound, significantly attenuates $\alpha \mathrm{S}$ disease manifestation [39]. In the present study, the neuroprotective effect of Nilo may also have a contribution in anti-ER therapeutic intervention like salubrinal.

We also propose that the mechanism outlined here might be generally applicable to other age-related neurodegenerative diseases. Proteostatic imbalance, including autophagy impairment, is mechanistically implicated in the pathogenesis of multiple neurodegenerative diseases. Thus, we hypothesize that similar oxidative stress-induced
c-Abl activation in other neurodegenerative diseases will also cause autophagy deficits. For example, c-Abl pathway is described as a potential therapeutic target in amyotrophic lateral sclerosis (ALS) [74], AD and PD [75]. Therefore, the c-Abl activation could be a general molecular defect for age-related neurodegeneration.

\section{Conclusions}

In conclusion, our findings using in vivo and in vitro models demonstrated that $\alpha \mathrm{S}$ pathology induced c-Abl activation leads to p53-depdendent mTOR activation and autophagy impairment. The pathological relevance of the pathway is shown by the fact that Nilo treatment delays disease in TgA53T mouse model of $\alpha$-synucleinopathy and ameliorate signs of autophagic deficits. Thus, we uncovered a previously unknown pathological mechanism linking with c-Abl/p53 activation, mTOR/AMPK/ULK1, and autophagy. Additional studies to define further the mechanistic details will facilitate deeper understanding of pathogenic events in neurodegenerative disease and lead to novel therapeutic approaches.

\section{Supplementary information}

Supplementary information accompanies this paper at https://doi.org/10. 1186/s13024-020-00364-w.

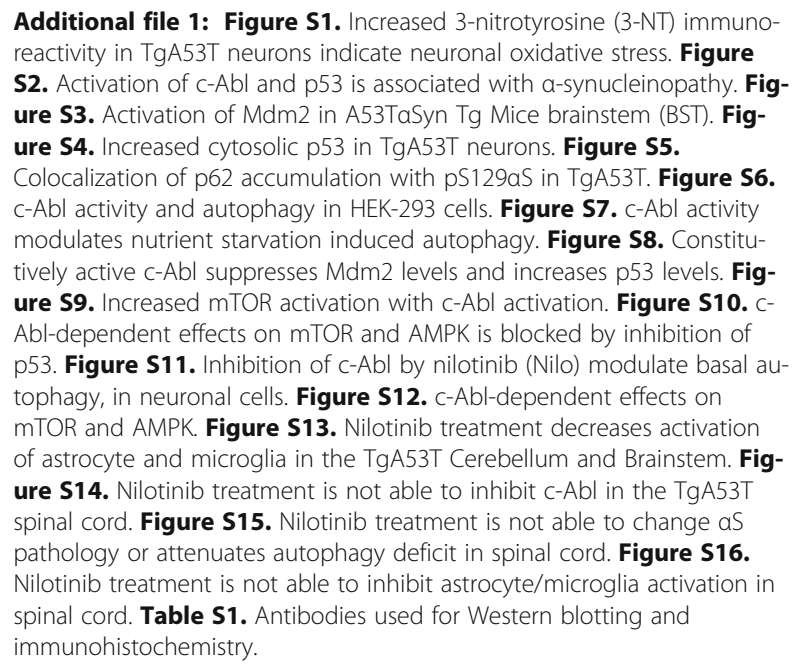
reactivity in TgA53T neurons indicate neuronal oxidative stress. Figure S2. Activation of c-Abl and p53 is associated with a-synucleinopathy. Figure S3. Activation of Mdm2 in A53TaSyn Tg Mice brainstem (BST). Figure S4. Increased cytosolic p53 in TgA53T neurons. Figure S5. Colocalization of p62 accumulation with pS129aS in TgA53T. Figure S6. c-Abl activity and autophagy in HEK-293 cells. Figure S7. c-Abl activity modulates nutrient starvation induced autophagy. Figure S8. Constitutively active c-Abl suppresses Mdm2 levels and increases p53 levels. Figure S9. Increased mTOR activation with c-Abl activation. Figure S10. cAbl-dependent effects on mTOR and AMPK is blocked by inhibition of p53. Figure S11. Inhibition of c-Abl by nilotinib (Nilo) modulate basal au tophagy, in neuronal cells. Figure S12. c-Abl-dependent effects on mTOR and AMPK. Figure S13. Nilotinib treatment decreases activation of astrocyte and microglia in the TgA53T Cerebellum and Brainstem. Figure S14. Nilotinib treatment is not able to inhibit c-Abl in the TgA53T spinal cord. Figure S15. Nilotinib treatment is not able to change aS pathology or attenuates autophagy deficit in spinal cord. Figure S16. Nilotinib treatment is not able to inhibit astrocyte/microglia activation in spinal cord. Table S1. Antibodies used for Western blotting and immunohistochemistry.

\section{Abbreviations}

3-NT: 3-nitrotyrosine; 4EBP1: Eukaryotic translation initiation factor 4E (elF4E)binding protein 1; Akt (PKB): protein kinase B; AMPK: adenosine monophosphate-activated protein kinase; Baf A1: bafilomycin A1;

BST: Brainstem; CTX: Cortex; DMEM: Dulbecco's modified Eagle's medium; DMSO: Dimethyl sulfoxide; EBSS: Earle's balanced salt solution; EV: Empty vector; GAPDH: glyceraldehyde 3-phosphate dehydrogenase; GFAP: Glial fibrillary acidic protein; Iba1: Ionized calcium binding adaptor molecule 1; LC3: microtubule-associated protein 1A/1B-light chain 3; Mdm2: Mouse double minute 2 homolog; mTOR: mammalian target of rapamycin; NeuN: Hexaribonucleotide Binding Protein-3; Nilo: Nilotinib; p62 (SQSTM1): sequestosome 1; PFT: Pifithrin-a; S6: ribosomal protein S6; SPC: Spinal Cord; ULK1: unc-51 like autophagy activating kinase 1; aS: asynuclein 


\section{Acknowledgements}

We thank and grateful to Cynthia Croy and Emilie Gasparini for excellent technical support, breeding and taking care of experimental animal. We are also grateful to Justin Barnes for preparing toxic lysates for experimentation.

\section{Authors' contributions}

MRK performed the data analysis, including the statistical analysis, and prepared the figures. MRK and EEL conducted in vivo study, and wrote the paper. MRK also designed and conducted entire in vitro experiments. JK made the initial in vivo observations. JM performed tissue processing and immunohistochemistry. HMM performed stereotaxic injections of lysates to establish the inoculation model. OP and JCT provided pathologically characterized human brain tissues. MKL supervised the project, including experimental design, data analysis, and writing of the manuscript. All authors read and approved the final manuscript.

\section{Funding}

This work was supported by grants from the National Institutes of Health (NIH) R01 NS038065, R01 NS086074, R01 NS092093, R01 NS108686, and R01 AG062135 (M.K.L.)

\section{Availability of data and materials}

The data and materials are available from corresponding author on reasonable request.

\section{Ethics approval}

All procedures for animal use were approved by the institutional Animal Care and Committee at the University of Minnesota.

\section{Consent for publication}

Not applicable.

\section{Competing interests}

The authors declare that they have no competing interests.

\section{Author details}

'Department of Neuroscience, University of Minnesota, Minneapolis, MN 55414, USA. ${ }^{2}$ Present Address: Department of Neural Development and Disease, Korea Brain Research Institute (KBRI), Daegu 41068, South Korea. ${ }^{3}$ Department of Pathology, Johns Hopkins University School of Medicine, Baltimore, MD 21287, USA. ${ }^{4}$ Institute for Translational Neuroscience, University of Minnesota, 2101 6th Street SE, Minneapolis, MN 55414, USA.

Received: 19 March 2019 Accepted: 13 February 2020

Published online: 16 April 2020

\section{References}

1. Jenner P, Olanow CW. The pathogenesis of cell death in Parkinson's disease. Neurology. 2006;66:S24-36

2. Lawson RA, McDonald C, Burn DJ. Defining delirium in idiopathic Parkinson's disease: a systematic review. Parkinsonism Relat Disord. 2018; S1353-8020(18):30421-8

3. Spillantini MG, Schmidt ML, Lee VM, Trojanowski JQ, Jakes R, Goedert M Alpha-synuclein in Lewy bodies. Nature. 1997;388(6645):839-40.

4. Goedert M, Spillantini MG, Del Tredici K, Braak H. 100 years of Lewy pathology. Nat Rev Neurol. 2013;9(1):13-24.

5. Obeso JA, Stamelou M, Goetz CG, Poewe W, Lang AE, Weintraub D, et al. Past, present, and future of parkinson's disease: a special essay on the 200th anniversary of the shaking palsy Mov Disord. Author manuscript; available in PMC 2018 Sep 1. Mov Disord. 2017;32(9):1264-310.

6. Klein C, Westenberger A. Genetics of Parkinson's disease. Cold Spring Harb Perspect Med. 2012. https://doi.org/10.1101/cshperspect.a008888.

7. Schneider SA, Alcalay RN. Neuropathology of genetic synucleinopathies with parkinsonism: review of the literature. Mov Disord. 2017:32(11):1504-23.

8. Jenner P. Oxidative stress in Parkinson's disease. Ann Neurol. 2003;53(Supp 3):S26-36

9. Hauser DN, Hastings TG. Mitochondrial dysfunction and oxidative stress in Parkinson's disease and monogenic parkinsonism. Neurobiol Dis. 2013;51: $35-42$.
10. Sison SL, Vermilyea SC, Emborg ME, Ebert AD. Using patient-derived induced pluripotent stem cells to identify Parkinson's disease-relevant phenotypes. Curr Neurol Neurosci Rep. 2018;18(12):84.

11. Kurtishi A, Rosen B, Patil KS, Alves GW, Møller SG. Cellular proteostasis in Neurodegeneration. Mol Neurobiol. 2018. https://doi.org/10.1007/s12035018-1334-z.

12. Klaips CL, Jayaraj GG, Hartl FU. Pathways of cellular proteostasis in aging and disease. J Cell Biol. 2018;217(1):51-63.

13. Brahmachari S, Karuppagounder SS, Ge P, Lee S, Dawson VL, Dawson TM, Ko HS. C-Abl and Parkinson's disease: mechanisms and therapeutic potential. J Park Dis. 2017;7(4):589-601.

14. Sun X, Wu F, Datta R, Kharbanda S, Kufe D. Interaction between protein kinase $\mathrm{C}$ delta and the $\mathrm{C}-\mathrm{Abl}$ tyrosine kinase in the cellular response to oxidative stress. J Biol Chem. 2000;275(11):7470-3.

15. Sun X, Majumder P, Shioya H, Wu F, Kumar S, Weichselbaum R, Kharbanda $S$, Kufe D. Activation of the cytoplasmic c-Abl tyrosine kinase by reactive oxygen species. J Biol Chem. 2000:275(23):17237-40.

16. Carr MI, Roderick JE, Zhang H, Woda BA, Kelliher MA, Jones SN Phosphorylation of the Mdm2 oncoprotein by the c-Abl tyrosine kinase regulates p53 tumor suppression and the radiosensitivity of mice. Proc Natl Acad Sci. 2016;113(52):15024-9.

17. Pendergast AM. The Abl family kinases: mechanisms of regulation and signaling. Adv Cancer Res. 2002:85:51-100.

18. Moresco EM, Koleske AJ. Regulation of neuronal morphogenesis and synaptic function by Abl family kinases. Curr Opin Neurobiol. 2003;13(5): $535-44$

19. Ko HS, Lee Y, Shin JH, Karuppagounder SS, Gadad BS, Koleske AJ, et al. Phosphorylation by the c-Abl protein tyrosine kinase inhibits parkin's ubiquitination and protective function. Proc Natl Acad Sci. 2010;107(38): 16691-6.

20. Imam SZ, Zhou Q, Yamamoto A, Valente AJ, Ali SF, Bains M, et al. Novel regulation of parkin function through c-Abl-mediated tyrosine phosphorylation: implications for Parkinson's disease. J Neurosci. 2011;31(1):157-63.

21. Lonskaya I, Hebron ML, Desforges NM, Franjie A, Moussa CE. Tyrosine kinase inhibition increases functional parkin-Beclin-1 interaction and enhances amyloid clearance and cognitive performance. EMBO Mol Med. 2013;5(8):1247-62.

22. Lonskaya I, Desforges NM, Hebron ML, Moussa CE. Ubiquitination increases parkin activity to promote autophagic a-synuclein clearance. PLoS One. 2013;8(12):e83914. https://doi.org/10.1371/journal.pone.0083914.

23. Hebron ML, Lonskaya I, Moussa CE. Tyrosine kinase inhibition facilitates autophagic SNCA/a-synuclein clearance. Autophagy. 2013;9(8):1249-50.

24. Brahmachari S, Ge P, Lee SH, Kim D, Karuppagounder SS, Kumar M, et al. Activation of tyrosine kinase c-Abl contributes to a-synuclein-induced neurodegeneration. J Clin Invest. 2016;126(8):2970-88.

25. Hebron ML, Lonskaya I, Moussa CE. Nilotinib reverses loss of dopamine neurons and improves motor behavior via autophagic degradation of asynuclein in Parkinson's disease models. Hum Mol Genet. 2013:22(16):3315-28.

26. Ren Y, Chen J, Wu X, Gui C, Mao K, Zou F, et al. Role of c-Abl-GSK3 $\beta$ signaling in MPP+-induced autophagy-Lysosomal dysfunction. Toxicol Sci. 2018;165(1):232-43.

27. Mahul-Mellier AL, Fauvet B, Gysbers A, Dikiy I, Oueslati A, Georgeon S, et al. C-Abl phosphorylates a-synuclein and regulates its degradation: implication for a-synuclein clearance and contribution to the pathogenesis of Parkinson's disease. Hum Mol Genet. 2014;23(11):2858-79.

28. Lee S, Kim S, Park YJ, Yun SP, Kwon SH, Kim D, et al. The c-Abl inhibitor, Radotinib $\mathrm{HCl}$, is neuroprotective in a preclinical Parkinson's disease mouse model. Hum Mol Genet. 2018;27(13):2344-56.

29. Dikiy I, Fauvet B, Jovičić A, Mahul-Mellier AL, Desobry C, El-Turk F, et al. Semisynthetic and in vitro phosphorylation of alpha-Synuclein at Y39 promotes functional partly helical membrane-bound states resembling those induced by PD mutations. ACS Chem Biol. 2016;11(9):2428-37.

30. Goldberg Z, Vogt Sionov R, Berger M, Zwang Y, Perets R, Van Etten RA, et al. Tyrosine phosphorylation of Mdm2 by c-Abl: implications for p53 regulation. EMBO J. 2002;21(14):3715-27.

31. Martin LJ, Pan Y, Price AC, Sterling W, Copeland NG, Jenkins NA, et al. Parkinson's disease alpha-synuclein transgenic mice develop neuronal mitochondrial degeneration and cell death. J Neurosci. 2006;26(1):41-50.

32. Lee MK, Stirling W, Xu Y, Xu X Qui D, Mandir AS, et al. Human alphasynuclein-harboring familial Parkinson's disease-linked Ala-53 --> Thr mutation causes neurodegenerative disease with alpha-synuclein aggregation in transgenic mice. Proc Natl Acad Sci. 2002;99(13):8968-73. 
33. Tasdemir E, Maiuri MC, Galluzzi L, Vitale I, Djavaheri-Mergny M, D'Amelio M, et al. Regulation of autophagy by cytoplasmic p53. Nat Cell Biol. 2008;10(6):676-87.

34. von Coelln R, Thomas B, Andrabi SA, Lim KL, Savitt JM, Saffary R, et al. Inclusion body formation and neurodegeneration are parkin independent in a mouse model of alpha-synucleinopathy. J Neurosci. 2006;26:3685-96.

35. Wang J, Martin E, Gonzales V, Borchelt DR, Lee MK. Differential regulation of small heat shock proteins in transgenic mouse models of neurodegenerative diseases. Neurobiol Aging. 2008;29(4):586-97.

36. Luk KC, Kehm VM, Zhang B, O'Brien P, Trojanowski JQ, Lee VM. Intracerebral inoculation of pathological alpha-synuclein initiates a rapidly progressive neurodegenerative alpha-synucleinopathy in mice. J Exp Med. 2012;209: 975-86.

37. Li W, Lesuisse $C, X u$ Y, Troncoso JC, Price DL, Lee MK. Stabilization of alphasynuclein protein with aging and familial parkinson's disease-linked A53T mutation. J Neurosci. 2004:24(33):7400-9.

38. Pletnikova O, West N, Lee MK, Rudow GL, Skolasky RL, Dawson TM, et al. Abeta deposition is associated with enhanced cortical alpha-synuclein lesions in Lewy body diseases. Neurobiol Aging. 2005;26:1183-92.

39. Colla E, Coune P, Liu Y, Pletnikova O, Troncoso JC, Iwatsubo T, et al. Endoplasmic reticulum stress is important for the manifestations of alphaSynucleinopathy in vivo. J Neurosci. 2012;32:3306-20.

40. Colla E, Jensen PH, Pletnikova O, Troncoso JC, Glabe C, Lee MK. Accumulation of toxic alpha-Synuclein oligomer within endoplasmic reticulum occurs in alpha-Synucleinopathy in vivo. J Neurosci. 2012;32:3301-5.

41. Liu Y, Yoo MJ, Savonenko A, Stirling W, Price DL, Borchelt DR, et al. Amyloid pathology is associated with progressive monoaminergic neurodegeneration in a transgenic mouse model of Alzheimer's disease. J Neurosci. 2008;28(51):13805-14

42. Zambrano N, Bruni P, Minopoli G, Mosca R, Molino D, Russo C, et al. The beta-amyloid precursor protein APP is tyrosine-phosphorylated in cells expressing a constitutively active form of the Abl protoncogene. J Biol Chem. 2001;276(23):19787-92.

43. Furstoss O, Dorey K, Simon V, Barilà D, Superti-Furga G, Roche S. C-Abl is an effector of Src for growth factor-induced c-myc expression and DNA synthesis. EMBO J. 2002;21(4):514-24.

44. Barilá D, Mangano R, Gonfloni S, Kretzschmar J, Moro M, Bohmann D, et al. A nuclear tyrosine phosphorylation circuit: C-Jun as an activator and substrate of c-Abl and JNK. EMBO J. 2000;19(2):273-81.

45. Karuppagounder SS, Brahmachari S, Lee Y, Dawson VL, Dawson TM, Ko HS. The c-Abl inhibitor, nilotinib, protects dopaminergic neurons in a preclinical animal model of Parkinson's disease. Sci Rep. 2014;4:4874.

46. Levine AJ, Hu W, Feng Z. The P53 pathway: what questions remain to be explored? Cell Death Differ. 2006;13(6):1027-36.

47. Darwish RS, Amiridze N, Aarabi B. Nitrotyrosine as an oxidative stress marker: evidence for involvement in neurologic outcome in human traumatic brain injury. J Trauma. 2007;63(2):439-42.

48. Karim MR, Kanazawa T, Daigaku Y, Fujimura S, Miotto G, Kadowaki M. Cytosolic LC3 ratio as a sensitive index of macroautophagy in isolated rat hepatocytes and H4-II-E cells. Autophagy. 2007:3(6):553-60.

49. Watanabe Y, Tatebe H, Taguchi K, Endo Y, Tokuda T, Mizuno T, et al. p62/ SQSTM1-dependent autophagy of Lewy body-like a-synuclein inclusions. PLoS One. 2012;7(12):e52868. https://doi.org/10.1371/journal.pone.0052868.

50. Jiang P, Mizushima N. LC3- and p62-based biochemical methods for the analysis of autophagy progression in mammalian cells. Methods. 2015;75:13-8.

51. Del Tredici K, Rub U, De Vos RA, Bohl JR, Braak H. Where does parkinson disease pathology begin in the brain? J Neuropathol Exp Neurol. 2000;61: 413-26.

52. de la Monte SM, Sohn YK, Ganju N, Wands JR. P53- and CD95-associated apoptosis in neurodegenerative diseases. Lab Investig. 1998;78(4):401-11.

53. Oueslati A, Schneider BL, Aebischer P, Lashuel HA. Polo-like kinase 2 regulates selective autophagic a-synuclein clearance and suppresses its toxicity in vivo. Proc Natl Acad Sci. 2013;110(41):E3945-54.

54. Sarkar S, Davies JE, Huang Z, Tunnacliffe A, Rubinsztein DC. Trehalose, a novel mTOR-independent autophagy enhancer, accelerates the clearance of mutant huntingtin and alpha-synuclein. J Biol Chem. 2007;282(8):5641-52.

55. Alers S, Löffler AS, Wesselborg S, Stork B. Role of AMPK-mTOR-UIk1/2 in the regulation of autophagy: cross talk, shortcuts, and feedbacks. Mol Cell Biol. 2012;32(1):2-11.

56. Egan DF, Shackelford DB, Mihaylova MM, Gelino S, Kohnz RA, Mair W, et al. Phosphorylation of ULK1 (hATG1) by AMP-activated protein kinase connects energy sensing to mitophagy. Science. 2011;331(6016):456-61.
57. Sarbassov DD, Ali SM, Sabatini DM. Growing roles for the mTOR pathway. Curr Opin Cell Biol. 2005;17(6):596-603.

58. Kim J, Kundu M, Viollet B, Guan KL. AMPK and mTOR regulate autophagy through direct phosphorylation of Ulk1. Nat Cell Biol. 2011;13(2):132-41.

59. Inoue K, Fry EA, Frazier DP. Transcription factors that interact with p53 and Mdm2. Int J Cancer. 2016;138(7):1577-85.

60. Levav-Cohen Y, Goldberg Z, Zuckerman V, Grossman T, Haupt S, Haupt Y. C-Abl as a modulator of p53. Biochem Biophys Res Commun. 2005;331(3):737-49.

61. Lohrum MA, Vousden $\mathrm{KH}$. Regulation and activation of p53 and its family members. Cell Death Differ. 1999;6(12):1162-8.

62. Sionov RV, Moallem E, Berger M, Kazaz A, Gerlitz O, Ben-Neriah Y, Oren M, Haupt Y. C-Abl neutralizes the inhibitory effect of Mdm2 on p53. J Biol Chem. 1999:274(13):8371-4.

63. Marine JC, Lozano G. Mdm2-mediated ubiquitylation: p53 and beyond. Cell Death Differ. 2010;17(1):93-102

64. Alves da Costa C, Checler F. Apoptosis in Parkinson's disease: is p53 the missing link between genetic and sporadic parkinsonism? Cell Signal. 2011;23(6):963-8.

65. Tatton WG, Chalmers-Redman RM, Elstner M, Leesch W, Jagodzinski FB, Stupak DP, et al. Glyceraldehyde-3-phosphate dehydrogenase in neurodegeneration and apoptosis signaling. J Neural Transm Suppl. 2000;60:77-100.

66. Karunakaran S, Saeed U, Mishra M, Valli RK, Joshi SD, Meka DP, et al. Selective activation of p38 mitogen-activated protein kinase in dopaminergic neurons of substantia nigra leads to nuclear translocation of p53 in 1-methyl-4-phenyl-1,2,3,6-tetrahydropyridine-treated mice. J Neurosci. 2008;28(47):12500-9.

67. White E. Autophagy and p53. Cold Spring Harb Perspect Med. 2016;6(4): a026120. https://doi.org/10.1101/cshperspect.a026120.

68. Tasdemir E, Chiara Maiuri M, Morselli E, Criollo A, D'Amelio M, DjavaheriMergny $\mathrm{M}$, et al. A dual role of p53 in the control of autophagy. Autophagy. 2008:4(6):810-4

69. Hoshino A, Ariyoshi M, Okawa Y, Kaimoto S, Uchihashi M, Fukai K, et al. Inhibition of p53 preserves Parkin-mediated mitophagy and pancreatic $\beta$ cell function in diabetes. Proc Natl Acad Sci. 2014:111(8):3116-21.

70. Neitemeier S, Ganjam GK, Diemert S, Culmsee C. Pifithrin-a provides neuroprotective effects at the level of mitochondria independently of p53 inhibition. Apoptosis. 2014;19(12):1665-77.

71. Lindholm D, Pham DD, Cascone A, Eriksson O, Wennerberg K, Saarma M. cAbl inhibitors enable insights into the pathophysiology and neuroprotection in Parkinson's disease. Front Aging Neurosci. 2016:8:254. eCollection 2016. https://doi.org/10.3389/fnagi.2016.00254.

72. Pagan F, Hebron M, Valadez EH, Torres-Yaghi Y, Huang X, Mills RR, Wilmarth BM, Howard H, Dunn C, Carlson A, Lawler A, Rogers SL, Falconer RA, Ahn J, Li Z, Moussa C. Nilotinib effects in Parkinson's disease and dementia with Lewy bodies. J Park Dis. 2016:6(3):503-17.

73. Pagan FL, Hebron ML, Wilmarth B, Torres-Yaghi Y, Lawler A, Mundel EE, Yusuf N, Starr NJ, Arellano J, Howard HH, Peyton M, Matar S, Liu X, Fowler AJ, Schwartz SL, Ahn J, Moussa C. Pharmacokinetics and pharmacodynamics of a single dose Nilotinib in individuals with Parkinson's disease. Pharmacol Res Perspect. 2019;7(2):e00470. https://doi.org/10.1002/prp2.470.

74. Imamura K, Izumi Y, Watanabe A, Tsukita K, Woltjen K, Yamamoto T, et al. The Src/c-Abl pathway is a potential therapeutic target in amyotrophic lateral sclerosis. Sci Transl Med. 2017;9(391):eaaf3962. https://doi.org/10. 1126/scitransImed.aaf3962.

75. Schlatterer SD, Acker CM, Davies P. c-Abl in neurodegenerative disease. J Mol Neurosci. 2011:45(3):445-52.

\section{Publisher's Note}

Springer Nature remains neutral with regard to jurisdictional claims in published maps and institutional affiliations. 